\title{
Sliding Paths for Series of Frenkel-Kontorova Models - A contribution to the concept of 1D-superlubricity
}

\author{
W. Quapp ${ }^{1}$ and J. M. Bofill ${ }^{2}$ \\ 1 Mathematisches Institut, Universität Leipzig, PF 100920, D-04009 Leipzig, Germany \\ 2 Departament de Química Inorgànica i Orgànica, Secció de Química Orgànica, and Institut de Química Teòrica i \\ Computacional, (IQTCUB), Universitat de Barcelona, Martí i Franquès 1, 08028 Barcelona, Spain
}

Received: April 08, 2019 / Revised version: June 18, 2019

\begin{abstract}
Newton trajectories are used to calculate low energy pathways for a series of Frenkel-Kontorova models with 6 and up to 69 particles thus up to a medium chain, and an expedition to 101 particles. The model is a finite chain with free-end boundary conditions. It has two competing potentials and an additional, external force. We optimize stationary structures and calculate the low energy paths between global minimums for a movement of the chain over its on-site potential, if an external tilting by a pushand/or pull direction is applied. We propose to understand a low energy path for a possibility of a superlubricity of the chain. We compare different misfit parameters. The result is that the minimums differ only little, however, the critical length of the chain, $N_{c r}$, depends on the misfit parameter. $N_{c r}$ describes the end of a 'good' calculability of the Newton trajectory which follows the low energy pathway of the chain through the potential energy surface, for a movement of the chain along the axis. We discuss reasons for the boundary of an $N_{c r}$. However, we assume that the low energy paths exist beyond their calculability by NTs.
\end{abstract}

PACS. Frenkel-Kontorova model - misfit parameter - critical length - tilting and sliding - Newton trajectory - superlubricity

\section{Introduction}

We select a subsystem on an axis in solitary confinement from a larger example of particles for solid-state Physics. The subsystem consists of interacting elements, and one considers the remaining part as a substrate. The substrate acts by a fixed potential on the extracted subsystem. One example of such a model is the Frenkel-Kontorova (FK) model $[1,2]$ introduced in 1938 by Yakov Il'ich Frenkel and Tatyana Kontorova. The subsystem is represented by a discrete chain of atoms harmonically coupled with their nearest neighbours, while the impact of the fixed substrate on the one-dimensional subsystem can be described by a sinusoidal form. It can be a standing wave of light, an optical lattice $[3,4]$, if the chain consists of ions. One supposes that the equilibrium distance associated with the inter-particle interaction may or may not coincide with the period of the substrate potential.

The concept has subsequently been involved in many applications of the model: tribology $[5,6]$ charge-density waves conductors [7-10], charge transport in solids and on crystal surfaces [11], magnetic or ferro- and antiferromagnetic domain walls [12], magnetic superlattices [13], superconductivity [14-16], vortex matter [17-19], fractal spin glasses [20], Josephson junctions [21,22], quantum al- gorithm [9], and H-bonded chains [1], just to name a few. Sliding friction forms a broad interdisciplinary field of interest that often involves the application of the FK model $[5,23,24]$ but for quite more particles than those which we treat here.

Here we understand static structural properties of this model of condensed matter. One can find recent applications of the Newton trajectory (NT) theory to the FK model in Refs. [25,26]. In many applications, one particularly interesting aspect of the FK model is its driven form. The FK model additionally exhibits a very rich behaviour when it is subjected to an external tilting force. NTs describe the curve of the force displaced stationary points (FDSPs) for every tilted potential energy surface (PES) under the increasing external force which is applied to move the chain over the substrate. NTs are mathematical tools. They are curves on the given PES where at every curve point the gradient of the PES points into the same direction called the search direction [27]. Of course, it is the direction of the external force. NTs are static properties of the geometry of the PES.

We compare here by means of NTs the FK models for $N=6$ to 101 particles with different relations of the two main parameters of the model, the natural spacing of the chain, $a_{0}$, and the periodicity of the on-site potential, 
$a_{s}$. We treat the values, $M F=a_{0} / a_{s}=(1,2 / 3,0.61803$, $1 / 2) . M F$ is named the misfit parameter. The value 1 is the 'trivial' one where every atom has its own well of the on-site potential, 2/3 is used in Ref. [28], 1/2 makes very symmetric structures of the chain $[29,30]$, and the value 0.61803 is the reciprocal golden mean, an often used value to demonstrate the "incommensurability" - especially for the FK model [31]. Compare also Ref.[25] for a theoretical reasoning. In contrast, we do not find large qualitative differences for stationary states of the PES. Low saddle points (SP) emerge for all values of the misfit parameter where $M F=1$ plays an extra role. For the calculation of NTs over the corresponding PESes of the FK model, we often use the pull- and push direction $(1,0, \ldots, 0,1)^{T}$ in $N$ dimensions. Thus, the first particle is pushed into the chain, but the last, the $N$ th particle is pulled off the chain. Then all other particles may move by the spring forces between them. The two edge-driven forces are given, but the internal movement of the chain is a black box. This direction is sometimes 'quite better' for NTs than others, like the only push direction, $(1,0, \ldots, 0)^{T}$, or the only-pull direction, $(0, \ldots, 0,1)^{T}$, or an equal force $[30,32]$ to all particles, the direction $(1,1, \ldots, 1,1)^{T}$, making a tilted washboard potential. 'Quite better' here means the property of the corresponding NT to find a minimum energy pathway (MEP) through the PES mountains, or at least a similar low energy path (LEP). Unfortunately, the only-pull version is often applied in atomic force microscopy experiments, and the full force is often applied for usual tribological experiments $[5,6]$, as well as for charge-density waves [7-11]. So, our result may be somewhat besides of current experimental results.

Given the case that an NT describes an MEP, or an LEP, as a deep valley with a low SP through the PES mountains, for a full movement of the chain over a period of the on-site potential, $a_{s}$. Then we propose to think about the path through the surrounding very higher PES mountains for a way where an external tilting force along the direction of the NT can move the chain with very low resistance of the on-site potential, thus, the direction is a 'lubricated' direction.

Note: the so called superlubricity [33-38] is the Holy Grail of tribology.

We propose to understand a low energy pathway of the PES for the full movement of the chain along a period of the on-site potential as a

\section{definition of 1D-superlubricity.}

As it is already known, superlubricity does not mean that we have zero resistance of the on-site potential. But if one can find a direction of the external force which uses the low energy path for the movement of the chain under a tilting then this may be named superlubricity. The special NT which describes the LEP delivers the tilting direction in the $N$-dimensional space of the chain. This NT is the key for the keyhole of the LEP. Note that the usually treated 'natural' direction $(1,1, \ldots, 1,1)^{T}$ is not the key! See subsection 5.4 below.

This paper is meant as a short, and certainly not exhaustive, review to more than 115 of such low energy paths. We try to determine an approximate number $N_{c r}$ of a critical chain length up to which a complete NT describes a successful tilting path. We use a model calculation where the spring forces of the chain and the substrate potential are equal (see equation (1) below with $v=k=1$ ). The push- and pull direction is best for small chains, but we also have to look around. Here the different $M F$ parameters come into play. In the case of no misfit between $a_{s}$ and $a_{0}$, so to say, the push- and pull direction does not work, at all, from the beginning of low dimensions. We have only critical dimensions $N$. And we do not get a really low energy path. For the next maximally symmetric case, $M F=1 / 2$, we obtain $N_{c r} \approx 20$, for $M F=2 / 3$, we get $N_{c r} \approx 30$, but for the reciprocal golden mean, $0.618034 \ldots$, we find $N_{c r} \approx 50$. There are nice complete, unique NTs for the low energy path for $N$ up to 64 , however, in between are gaps for single numbers $N$ where we did not find a unique, useful NT. We discuss different reasons why we can lose the 'goodness' of an NT over the PES.

The paper has the following Sections. In Section 2 we report on the FK model which is additionally tilted. A short review of the theory of NTs is added in Section 3 and it is explained how NTs can be applied to the FK model. Beginning with Section 4 we enrol a gallery of examples for different misfit parameters, and different small chains with 6 to 101 particles. For $M F=2 / 3$, the cases $N=2$ to $N=5$, and $N=23$ particles are discussed elsewhere $[25,26]$. Here we first continue this case $M F=2 / 3$ in Section 4 . We get symmetric and asymmetric minimums and saddle points (SPs) of the PESes [39], as well as a full continuation of the existence of the LEPs for all $N$. The misfit parameter $1 / 2$ is discussed in Section 5. In Section 6 we treat the reciprocal golden mean which we push along the series to 69 atoms, and calculate a runaway of 101 atoms. The last case of study is the case of no misfit, thus $M F=1$, in Section 7. A comparison of the different cases is done in Section 8. A Discussion in Section 9 and a Conclusion in Section 10 form the end of the paper. Data of the discussed cases are collected in diverse Supplementary Materials.

\section{The FK model}

\subsection{Model formula}

$\mathbf{x}=\left(x_{1}, \ldots, x_{N}\right)$ is a linear chain of $N$ discrete atoms. The positions $x_{i}$ are on an axis. For all atoms holds $x_{i}<x_{i+1}$. They are sorted in a fixed order. We treat a finite chain, thus $N$ is less than infinity [40-47], it goes up to 101 .

A spring force acts with a force constant $k$ between the atoms which results in a constant natural distance $a_{o}$ of the atoms. Without the side force, the end points of the chain are such that the average distance is $a_{o}=$ 
$\left(x_{N}-x_{1}\right) /(N-1)$. A fixed on-site potential with a periodicity, $a_{s}$, acts on the atoms in concurrence with the springs between them. The on-site potential mimics a rigid nondeformable substrate. The ratio $a_{o} / a_{s}$ is named the misfit parameter. The PES for the variable changes of the $x_{i}$ is the Frenkel-Kontorova model

$V(\mathbf{x})=v \sum_{i=1}^{N}\left(1-\cos \left(\frac{2 \pi x_{i}}{a_{s}}\right)\right)+\sum_{i=1}^{N-1} \frac{k}{2}\left(x_{i+1}-x_{i}-a_{o}\right)^{2}$.

$v$ is the corrugation parameter of the on-site potential. Here we put the factor at the sinusoidal potential, $v=1$, as well as the spring constant, $k=1$, throughout the paper. The ratio of the strength of the on-site potential to that of the spring potential is 1 . Because $v>0$, the on-site potential will modulate the chain [48], and we will generally get another average spacing, $\tilde{a}_{o}$. All quantities referred to in this work are dimensionless. Derivations of the PES (1) are reported elsewhere [25]. The calculation of stationary points of the FK model is done by the application of NTs. For control reasons, we also use the optimization in Mathematica.

\subsection{Tilting of the FK model}

Additionally to the two forces of the FK model, we use an external, linear force in the ansatz [1,2,28,30,43,49-57]. We name the resulting PES an effective PES

$$
V_{F}(\mathbf{x})=V(\mathbf{x})-F\left(l_{1}, . ., l_{N}\right)^{T} \cdot \mathbf{x} .
$$

The multiplication point between the $N$-dimensional normalized force direction vector $\left(l_{1}, . ., l_{N}\right)^{T}$ and the $N$-variable $\mathbf{x}$ means the scalar product. $F$ is the factor for the amount of the force. The new term with the force $F$ is often named dc driving (dc: direct current) $[2,58]$. In many applications it is further subdivided in the dc-part, $F_{d c}$, and an ac-part (alternating current) $[30,32]$. It is $F_{a c} \cos (c t)$ with a constant $c$ and a time variable $t$.

The force tilts the former on-site potential with the incline $F$. If $F=0$, then a minimum structure for the chain will exist. But if all $l_{i}>0$ and $F$ is large enough there a minimum does not exit, at all $[25,43]$. It means that no global pinning can take place. Interesting is the so-called pinning-depinning transition, as well as the backward process [44].

For computational reasons we can use any directional vector, $\mathbf{l}=\left(l_{1}, \ldots, l_{N}\right)^{T}$ with $|l|=1$, of the $N$-dimensional coordinate space for a search along a special NT. This we will really do, see the next Section 3, and the applications below.

\section{Newton trajectories}

\subsection{The Definition}

The stationary points on the effective potential with the general force vector $\mathbf{f}=F\left(l_{1}, . ., l_{N}\right)^{T}$ satisfy the condition $\nabla_{\mathbf{x}} V_{F}(\mathbf{x})=\mathbf{0}$. Its minimums and SPs satisfy the vector equation

$$
\nabla_{\mathbf{x}} V_{F}(\mathbf{x})=\mathbf{g}(\mathbf{x})-\mathbf{f}=\mathbf{0} .
$$

One searches a point where the gradient of the original PES, $\mathbf{g}(\mathbf{x})$, has to be equal to the force, $\mathbf{f}$. Such an ansatz is named Newton trajectory $[59,60]$ to the force, $\mathbf{f}$. The gradient of $V(\mathbf{x})$ is the inner force of the chain, $\mathbf{x}$, against changes of its structure. This force has to be equal to the external force, $\mathbf{f}$. Then the new chain is again in an equilibrium. We are at a stationary point of $V_{F}(\mathbf{x})$. The NT describes a curve of force-displaced stationary points (FDSPs) of the tilted PES under a different load, $F[27$, 59-64]. Usually, the energy of minimums is increased, but the energy of SPs is lowered. This means that the barriers become lower. A very nice property of every effective PES (2) is that the NT to the search direction, $\mathbf{f}$, is the same on all $V_{F}$ if the directional vector, $\mathbf{l}$, is fixed.

Of course, an NT can be treated without the treatment of the physics of the external force in equation (2). One only needs an abstract search direction. Then any NT describes a connection between different stationary points of an index difference of one [65]. Following numerically an NT is a method to search a next SP if a minimum is given, or vice versa. Equation (3) can be written in a projector form $[59,60]$

$$
\left(\mathbf{U}-\mathbf{l}^{T}\right) \mathbf{g}(\mathbf{x})=\mathbf{0}
$$

where $\mathbf{U}$ is the unit matrix and the l-unit vector is the normalized direction of $\mathbf{f}$. Equation (4) means nothing else than that $\mathbf{g}$ and $\mathbf{l}$ are parallel. If we differentiate the projector equation (4) with respect to the parameter that characterizes the FDSPs curve, $s$ in $\mathbf{x}(s)$, we obtain with the Hessian, H, [60,62]

$$
\left(\mathbf{U}-1 \mathbf{l}^{T}\right) \mathbf{H}(\mathbf{x}) \frac{d \mathbf{x}}{d s}=\mathbf{0} .
$$

This is an expression of the tangent of the FDSPs curve. For the calculation, the continuous NT is approximated by $L$ node points. They should not be interchanged with the $N$ atoms of the chain. The $N$ atoms form a point in the $N$-dimensional configuration space. A curve of such points is the NT. And it is numerically treated by its $L$ nodes. Because we have the Hessian of the FK model at hand [25] then equation (5) is a way to generate the NT of a successive tilting. We use for the calculations a predictorcorrector method. For the predictor we use the tangent of the NT with equation (5).

\subsection{Application of NTs to the FK model}

We search for a minimum of the FK model in three steps: (1) We build a chain with spacing $a_{o}$. Set, for example, $x_{1}=0$ for the initial atom, so it is in the first well of the on-site potential, and set $x_{i}=(i-1) a_{o}$ for $i=2, . ., N$.

(2) We put the natural chain into the PES of equation (1), $V(\mathbf{x})$, and calculate the gradient $\mathbf{g}(\mathbf{x})$ [25].

(3) We form the normalized direction 1 along the negative gradient. It is the search direction of an NT which 

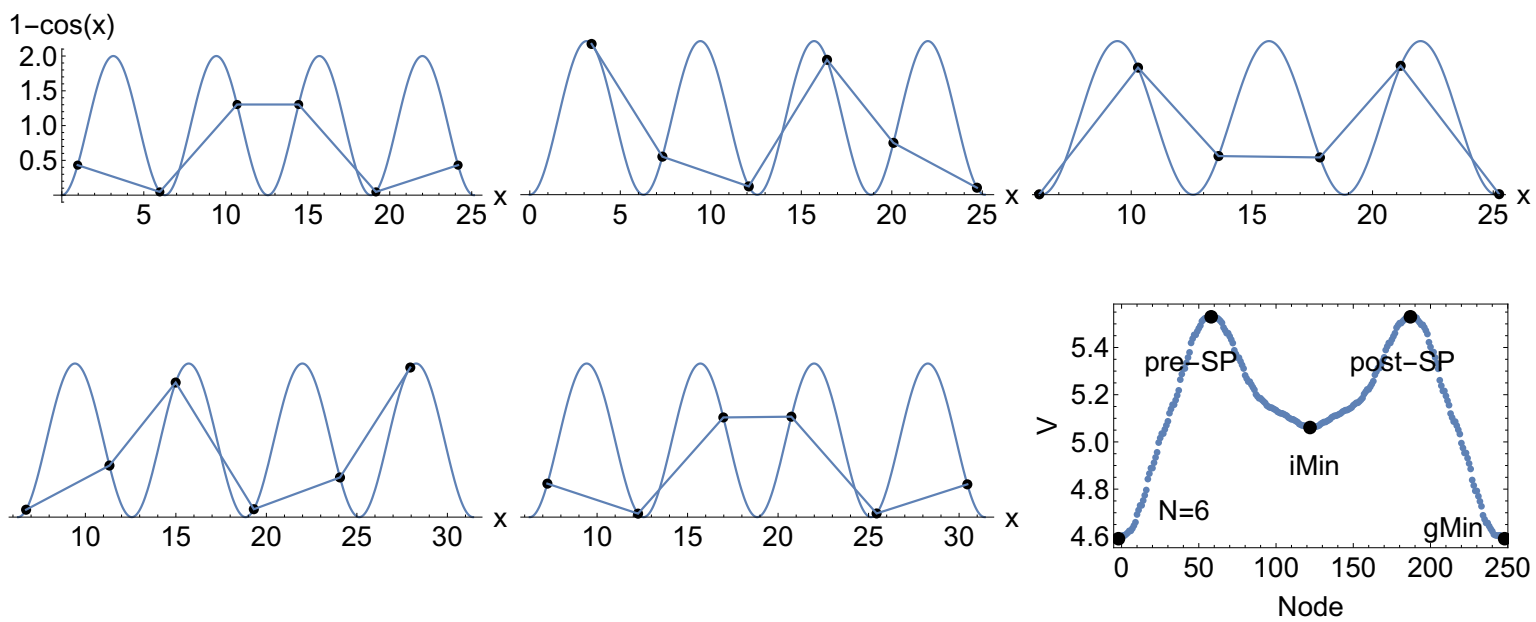

Fig. 1. Chain with 6 particles and misfit parameter $2 / 3$. Top line: schematic picture of the global minimum at the left panel, the Pre-SP at centre panel, and the right panel shows the intermediate minimum. For a better imagination, the particles of the linear chain are artificially set on the curve of the on-site potential, 1-cos(x). Bottom line: the post-SP and the next global minimum which is moved by $2 \pi$ on the axis. The right panel shows the profile of the energy over the MEP along the NT to direction $(1,0,0,0,0,1)^{T}$. The calculation is continuously done from the start left to finish at the right hand side. The global minimum is bottom left, then the pre-SP is surmounted, and at the centre the intermediate minimum emerges. The NT goes further to the post-SP, and finishes at the next global minimum. The stationary states are depicted by black bullets.

goes through the surface point $V(\mathbf{x})$. We follow the NT downhill to the next stationary state which usually is a minimum. However, sometimes it is not the global minimum, but only an intermediate, in contrast to a conjecture in Ref. [66].

Such a minimum will always exist [67]. Each of the $N$ atoms of the chain will 'look' into (at least) one minimum well of the on-site potential. In the trivial case that $a_{o}=a_{s}$ one is finished. The natural chain is the global minimum. Usually, it is $a_{o} \neq a_{s}$. Then the minimization of the PES, equation (1), will move the atoms a little into their corresponding wells of the on-site potential down to an equilibrium with the spring forces, and the average spacing will become a value $\tilde{a}_{o}$ near the former $a_{o}$ (if $k>0$ what we always assume). The natural minimization excludes the assumption of periodic boundary conditions $[21,44]$

$$
x_{N}=x_{1}+2 a_{s} M
$$

with an appropriate integer $M$. The latter ansatz will fix $a_{o}$. However, in experiments with several tens of ions in a chain an inhomogeneous spacing was observed, in particular at the edges of the crystal [68]. But of course, the free ends of the chain are locked by the springs.

The boundary conditions are important for the global minimums of the chain. The periodic boundary conditions are often used, insteed of the free ones. Other boundary conditions change the global minimums of the chain. Why periodic boundary conditions are used? They allow to handle an 'infinite' chain $[69,70]$, they are a trick to treat an 'infinite' chain using a calculable finite part of the chain. However, in this paper we treat a finite chain from the be- ginning. Thus we can leave the boundary conditions free.

If some of the atoms, say $x_{j}$ for some $j$, are at the beginning on top of the on-site potential, then the other atoms left, or right from $x_{j}$ will pull them down to one of the both wells below the top. In some special cases one can finish at a symmetric SP, for example, if $a_{0}=a_{s} / 2$. Here one can give to one, or two, or all distances $a_{o}$ a tiny distortion. Then the NT finds the way downhill to the minimum.

The minimum is a point in the $N$-dimensional coordinate space. If the chain is here, it is fixed if no other outer force moves it out of its state. No sliding or so can happen. The same applies if $N$ becomes larger and larger, compare Ref.[71]. Because each of the $N$ atoms sits in its own minimum well of the on-site potential. And so each of the atoms must be moved out of the global minimum for a hypothetical sliding.

\section{Examples of FK chains for commensurate misfit $2 / 3$}

\subsection{Low Dimensions}

First we use $a_{s}=2 \pi, a_{0}=4 \pi / 3, v=k=1$, thus the misfit parameter $M F$ is $2 / 3$. The case $N=6$ can be explained by Figure 1. Starting at the left global minimum, one can come to the 'pre-SP' of Figure 1 by moving the particles 1 and 4 to the right hand side over the peaks of their onsite potential. It is the pathway along the NT to direction $(1,0,0,0,0,1)^{T}$. In the next step, the SP structure can relax by a movement by all particles to the right hand side, 


\begin{tabular}{|c|cccc|c|}
\hline $\mathrm{N}$ & gMin & iMin & SP $_{1}$ & barrier & hMin $^{* *}$ \\
\hline 6 & 4.603 & 5.062 & 5.532 & 0.929 & \\
7 & 5.336 & 6.157 & 6.607 & 1.271 & \\
8 & 6.240 & $7.601^{*}$ & 7.674 & 1.434 & \\
9 & 7.250 & 7.671 & 8.272 & 1.022 & \\
10 & 8.040 & 8.609 & 9.262 & 1.222 & \\
11 & 8.969 & $10.221^{*}$ & 10.373 & 1.404 & \\
12 & 9.937 & $10.315^{*}$ & 10.994 & 1.057 & 11.872 \\
13 & 10.748 & 11.210 & 11.957 & 1.209 & \\
14 & 11.687 & 12.639 & 13.044 & 1.357 & \\
15 & 12.639 & $12.992^{*}$ & 13.710 & 1.071 & 14.269 \\
16 & 13.459 & 13.841 & 14.659 & 1.2 & \\
17 & 14.400 & 15.345 & 15.750 & 1.35 & \\
18 & 15.347 & 15.679 & 16.422 & 1.075 & 16.677 \\
19 & 16.170 & $16.514^{*}$ & 17.368 & 1.198 & 18.311 \\
20 & 17.112 & 17.986 & 18.459 & 1.347 & \\
\hline
\end{tabular}

\begin{tabular}{|c|cccc|}
\hline $\mathrm{N}$ & gMin & iMin $^{*}$ & $\mathrm{SP}_{1}$ & barrier \\
\hline 21 & 18.059 & 18.38 & 19.134 & 1.076 \\
22 & 18.883 & 19.196 & 20.069 & 1.189 \\
23 & 19.825 & 20.656 & 21.17 & 1.345 \\
24 & 20.769 & 21.082 & 21.846 & 1.076 \\
25 & 21.593 & 21.892 & 22.790 & 1.197 \\
26 & 22.536 & 23.339 & 23.881 & 1.345 \\
27 & 23.479 & 23.789 & 24.557 & 1.078 \\
28 & 24.304 & 24.591 & 25.501 & 1.197 \\
29 & 25.247 & 26.024 & 26.592 & 1.345 \\
30 & 26.191 & 26.499 & 27.269 & 1.078 \\
31 & 27.015 & 27.304 & 28.212 & 1.196 \\
32 & 27.959 & 28.722 & 29.299 & 1.341 \\
33 & 28.902 & 29.209 & 30.393 & $1.491^{\#}$ \\
34 & 29.727 & 30.018 & 30.928 & 1.201 \\
35 & 30.67 & 31.426 & 32.016 & 1.345 \\
\hline
\end{tabular}

Table 1. Energies of the $N$-chains for misfit parameter $2 / 3$ at stationary points. gMin is the global minimum, iMin is the intermediate on the LEP, $\mathrm{SP}_{1}$ is the saddle in between. ${ }^{*} \mathrm{It}$ is a flat double minimum. **Here a higher energy minimum, hMin, outside the MEP is known. ${ }^{\#}$ The barrier is too high, in comparison to other $N$. The second possibility with two NTs, one part pull-only, the other part push-only goes over a still higher barrier of 1.567 . Note that the barriers can generally be somewhat over the lowest possible value if another MEP exists there, compare the case $N=23$ in Ref.[26]. If we had found 'any' LEP, we would have stopped the calculation.

forming the intermediate minimum of Figure 1, top right panel. It is compressed by nearly $2 \pi$ against the global minimum, thus, its energy is higher. It can be seen in Figure 1 in the right bottom panel for the intermediate minimum (iMin) by the black bullet in the centre of the curve, at node 125 . The next two steps are again a movement over a 'post-SP' with a mirror structure to the given pre-SP, and a next movement to an analogous global minimum, but moved by $2 \pi$ to the right hand side on the axis, see Figure 1. Though the $N=6$ case is simple we discuss it with Scheme 1 for the wells of the on-site potential.

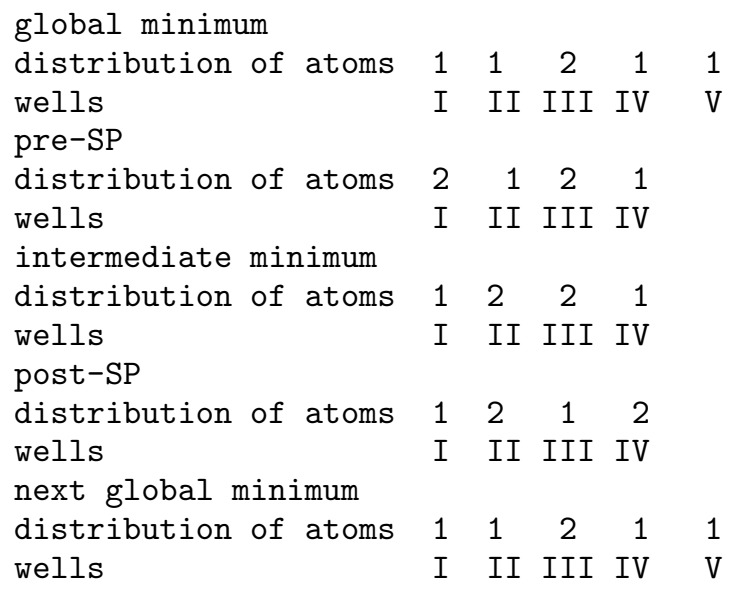

Scheme 1: Distribution of the 6 atoms of the chain into the wells of the $1-\cos (\mathrm{x})$ potential.

The saddles as well as the intermediate structure are compressed. They need one well less. At the pre-SP, the atoms 1 and 4 move into the next well, and the first two atoms form an anti-kink in the new first well which was before the second well. For the intermediate, the 4 internal atoms then form two neighbouring anti-kinks. They need more energy than the ground state. The post-SP is the mirror picture of the pre-SP: here the last two atoms form the anti-kink.

In Table 1 we report the data of minimums, SPs, and intermediates, iMin, on a full pathway through the PES mountains along the corresponding NT to direction $(1,0, \ldots, 0,1)^{T}$. The path always starts with $\mathrm{x}_{1}$ in the valley of the on-site potential near zero, and ends with $\mathrm{x}_{1}$ moved by $2 \pi$, the parameter $a_{s}$ of the on-site potential. Along the path, the energy has to be lower than the energy of the $\mathrm{SP}_{1}$. It has to be surmounted. Then the full chain is moved by approximate $a_{s}$ along the NT. The existence of such a path does not mean that it is the MEP over the lowest possible SPs through the mountains, as was already obtained in the case $N=23$, discussed elsewhere [26], but it should be an LEP with $\mathrm{SP}_{1}$ energy near the other MEP. The iMin can be a compressed structure, or, contrary, a stretched structure, compare again the case $N=23$ in Ref. [26]. In the cases $N=17$ and $N=20$, other lower iMin exist. One could assume that a better LEP will emerge there as well. But in this case if then it will belong to a more complicated search direction of the corresponding NT. The low energy barrier of all cases in Table 1, for a movement of the full chain along its axis, comes from the opposite forces which different particles experience [25]. If one particle has to overcome the next peak of the onsite potential then another particle before it, or after it, slides down the slope of its well of the on-site potential. Of course, this property depends on the overall density of the particles of the chain, thus on the misfit parameter. But note: we never found a case where the lattice forces compensate totally. We always found a remainder of an amount to overcome the low $\mathrm{SP}_{1}$ of our low energy pathways on the PES of equation (1). It is in contrast to a 


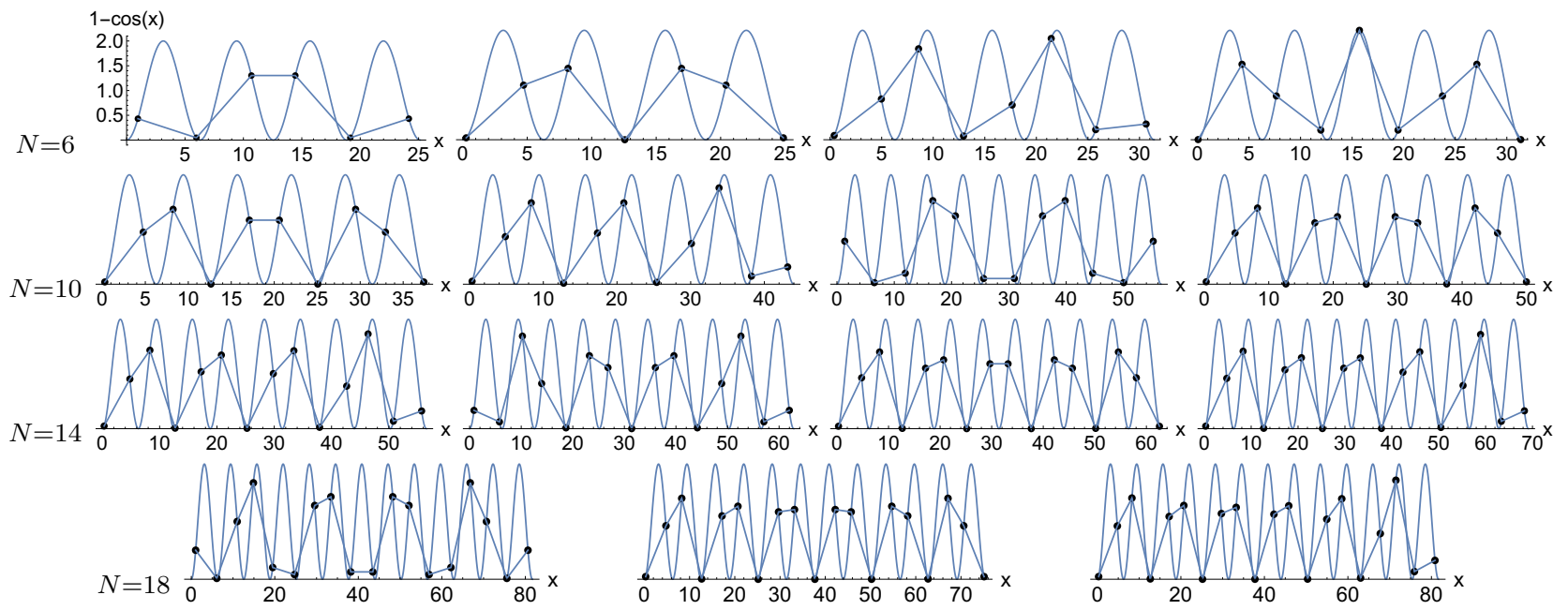

Fig. 2. Schematic picture of the global minimum structures for misfit parameter $2 / 3$ up to $N=20$.

special statement of Ref. [3] "that friction force on a twoion system could be made vanished by placing the two ions at positions where they experience opposite lattice forces."

In Figure 2 we represent the global minimums of the chains from $N=6$ to 20 . The energy profiles of NTs which start in these minimums to the used push- and pull direction $(1,0, \ldots, 0,1)^{T}$ are given in Figure 3 .

The ground state structures, as well as the energy profiles over the NTs to direction $(1,0, \ldots, 0,1)^{T}$, follow a trend modulo 3 with $N$. The ground states are symmetric for $N \equiv 0,1 \bmod 3$, where they are asymmetric for $N \equiv 2$ $\bmod 3$. Of course then a corresponding asymmetric mirror structure still exists here. The 'modulo'-trend depends on the simple misfit parameter of $2 / 3$ used here. The higher the number of particles in the chain is the more turning points (TPs) emerge for the corresponding NTs. TPs are points at the slope of the PES mountains where the NT changes its way from uphill to downhill, or vice versa [59]. But the more rugged the PES becomes, for higher $N$, the more TPs emerge. It becomes more dramatic in the next Subsection.

\subsection{Chains with critical length}

We further use the misfit parameter $2 / 3$. Figure 4 depicts the profiles over the NTs to the push- and pull direction. Here the TP problems of some NTs strengthen. Many TPs cause numerical problems for $N=28$. The given NT could only be gotten by some tests with the parameters of the programme.

However, for $N=30$, the diverse parts of the NT go far over the height of the $\mathrm{SP}_{1}$. This $\mathrm{NT}$ will become unusable for the push- and pull force for a driving. Note that NTs describe the way of moving stationary points under an external force. So $N=30$ may be the first critical length of the FK model with $M F=2 / 3$ with a breakdown of the 'superlubricity', compare Ref. [72]. In the panel for the case
$N=30$ in Figure 4, an NT emerges which loses the property to be a good device for a tilting: here a TP exists with an energy which is somewhat higher than the next SP. It means that the corresponding tilting moves together with the former minimum and the former $\mathrm{SP}_{1}$ on an higher energy than the $\mathrm{SP}_{1}$ itself had before. Such a transition is possibly inhibited, but not enforced, by the tilting. We try to understand what causes the strong swing in the energy. Like in the simple case $N=6$ above, we treat the distribution of the atoms in the wells of the $1-\cos (\mathrm{x})$ potential in Scheme 2 for different structures of the chain along the NT in the left side of panel $N=30$ up to iMin.

The ground state of the case $N=30$ is symmetric, the atoms are distributed into 21 wells with a regular pattern, see Figure 5. At a low energy left before the left maximal TP of the profile, the atom 19 of well XIII moves over the right top. It is depicted in line 2 of Scheme 2 by the symbol $1 / 2$ which means that the atom is distributed to both wells, left and right from the top of the on-site potential, to wells XIII and XIV. Then the NT increases to the maximal TP, shown in the next row. Here the top atom has moved back to the wells XII and XIII, however, at the left edge, we find two times two atoms in one well. This is a very energetic anti-kink. It makes that the number of wells, which the chain occupies, is reduced to XX. It may explain the high energy of the TP. If the NT relaxes from this TP, the anti-kink at the left hand side resolves, but the top atom moves to wells XI and XII. This top atom is now the number 16 in the chain. Then the NT again increases up to the pre-SP ${ }_{1}$ of the PES. It is again a quite regular structure, however asymmetric, with the first atom in a near top position. This may cause the high energy of the $\mathrm{SP}_{1}$. Further relaxation gives the intermediate minimum, the last line in Scheme 2. The top position in the wells XI and XII is now held by atom 17 . This top may cause the relative higher energy of the iMin in comparison to the global minimum. All in all, one may speculate that the instable anti-kink at the maximal energy TP could destroy the 'goodness' of the NT to serve as a leading line 

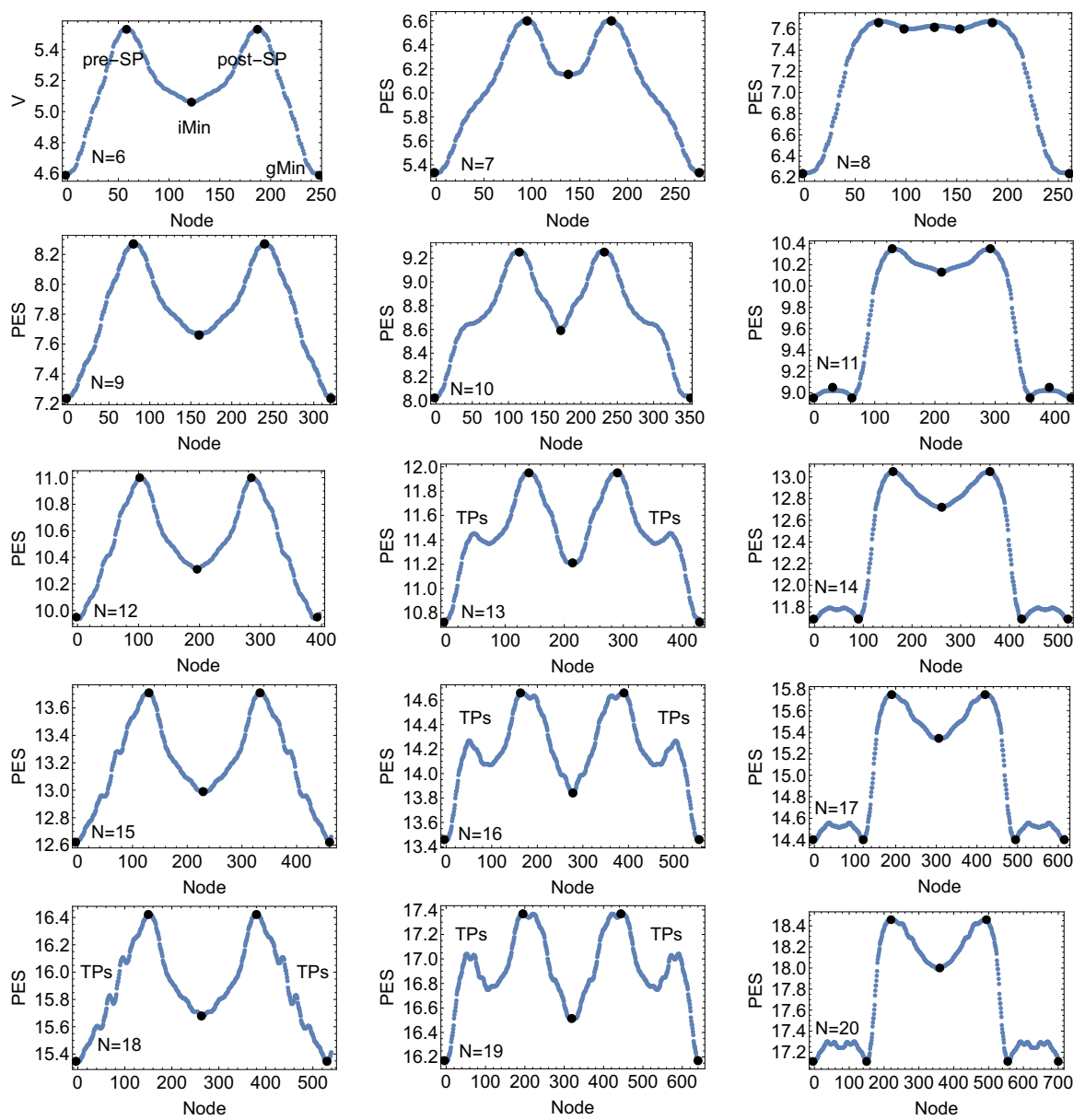

Fig. 3. Misfit parameter 2/3. Energy profiles over the NTs to direction $(1,0, \ldots, 0,1)^{T}$. The particles of the chain range from 6 to 20. Black bullets depict stationary points of the PES like minimums and SPs of index 1; however, other peaks are turning points of the NT.

for a tilting. The instable anti-kinks of the atoms 1 to 4 at the maximal TP are a kind of traffic jam [28] of the atoms of the chain on their way along the axis caused by the tilting along direction $(1,0, \ldots, 0,1)^{T}$. One may speculate that such a traffic jam is the beginning of the end of the superlubricity of the chain, at least for the used tilting direction. The cases $N=31$ to 35 show many small peaks from 'local' TPs. The case $N=33$ has a multitude of flat SPs of index one, beside the 'usual' SPs with an ordinary barrier. The NT to push- and pull direction does not seem to follow a single LEP, however, it splinters to a pathway over different valleys. Additionally, the first pre-SP $\mathrm{S}_{1}$ is too high, in comparison to other barriers. We guess that the NT does not follow an MEP, and that this search direction comes to its final possibility here, at least for use as a tilting direction.

Note that the usual LEP consists of four parts: gMin to pre-SP $\mathrm{SP}_{1}$, pre-SP $\mathrm{SP}_{1}$ to iMin, iMin to post-SP $\mathrm{SP}_{1}$, and post-SP $\mathrm{SP}_{1}$ to the new gMin which is moved by $a_{s}$ on its axis. In the most general case, one can assume that 'good' NTs which follow the four valley grounds, have four different searchdirections. Here, up to $N=32$, we have one and the same search-direction for all four parts of the LEP of interest. It is a very special property of our kind of LEPs.

A second possibility for $N=33$ is shown in an additional panel in the last line of Figure 4 . We used for the calculation two different directions: pull-only, or push-only. One part up to the central iMin is the pull-only NT, the other part the push-only NT. Again we get a pathway which does not correspond to the pattern of another chain length. Again further intermediate minimums emerge. The steepest descent from the global $\mathrm{SP}_{1}$ at node 4620 is connected to the central iMin, and to the 'pre-iMin' structure with SP and minimum at nodes 1592 and 1722. However, both profiles are quite symmetric and if we combine both NTs then the path leads to a correct movement from the first global minimum structure to an $a_{s}=2 \pi$ moved analogous structure.

Another bad result we get in case $N=34$. The NT to the push- and pull direction climbs up a maximal TP 
which is much higher than the usual SP of index one on the LEP. The height of such TPs may forbid the use of the push- and pull direction for a tilting, though we assume that the corresponding LEP exists through the PES. In case $N=34$ we find a further speciality: near the nodes 2500, and 15000 in the panel two additional SPs emerge. They are flat SPs near a shoulder, in the promontory region. The obtained NT can be accepted if we sort these two SPs as shoulders [26]. A connection of two SPs of index one is forbidden for regular NTs by the NT theory.

The problem becomes still more complicated with the next case, $N=35$. Here the NT to the push- and pull direction turns after the first $\mathrm{SP}_{1}$ somewhat uphill and leads over two further SPs, one of index 2 , one of index 1 , before reaching at least the region of the iMin. In contrast, the steepest descent from the first pre-SP $\mathrm{S}_{1}$ leads directly to the iMin. It is proof that a correct LEP exists, however, the used NT cannot directly follow it. We can compare the value of the chain length with the definition of a critical $N_{c r}$-value in Refs. [72,73]. Different parameters for the FK model are used here, thus the critical $N_{c r}$ becomes quite higher in those references. We may assume that a critical

\begin{tabular}{|c|ccc|}
\hline $\mathrm{N}$ & gMin & $\mathrm{SP}_{1}$ & barrier \\
\hline 7 & 5.6929 & 7.2106 & 1.5177 \\
8 & 6.608 & 7.9023 & 1.2943 \\
9 & 7.463 & 8.8964 & 1.4334 \\
10 & 8.3546 & 9.7071 & 1.3525 \\
11 & 9.2235 & 10.629 & 1.4055 \\
12 & 10.1063 & 11.4798 & 1.3735 \\
13 & 10.9814 & 12.3747 & 1.3942 \\
14 & 11.86 & 13.244 & 1.384 \\
15 & 12.7371 & 14.1257 & 1.3888 \\
16 & 13.6145 & 14.9988 & 1.3843 \\
17 & 14.4936 & 15.8786 & 1.385 \\
18 & 15.3751 & 16.7546 & 1.3795 \\
19 & 16.2483 & 17.6336 & $1.3853^{*}$ \\
20 & 17.1243 & 18.5099 & 1.3856 \\
\hline
\end{tabular}

Table 2. Energies of some $N$-chains for misfit parameter $1 / 2$ at stationary points, and the barrier. *Beginning with the case $N=19$, the former 'cape' becomes higher than the path over the former iMin, thus we get here a global barrier of 1.751 over the $\mathrm{SP}_{1}$ of the cape, compare Figure 8, last panel.

SPs of index one, are reported in Table 2.

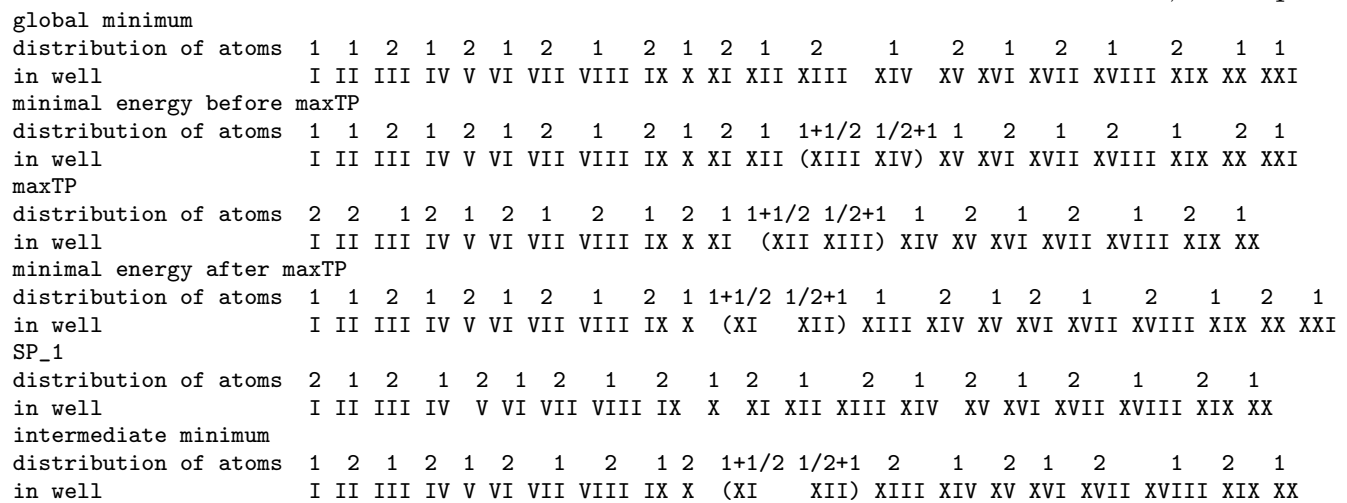

Scheme 2: Distribution of 30 atoms of the chain into the wells of the 1-cos(x) potential.

The distribution in the first and in the last line is symmetric, but asymmetric in the other lines.

critical $N_{c r}$ for misfit parameter $3 / 4$ is around 30 to 35 . The emergence of an NT with higher TPs than the correct $\mathrm{SP}_{1}$ of the LEP may destroy its model character of a good tilting path for a movement of the chain through the PES along a period of the on-site potential. In many cases one can assume that the usual LEP exists but the push- and pull direction may not be the appropriate direction for a tilting.

\section{Comparison for FK Chains with another misfit parameter}

We further use $v=k=1$ in equation (1). In this Section we test the commensurate case, $M F=1 / 2$, and in the next Section we study the often celebrated incommensurate reciprocal golden mean, $M F=2 /(1+\sqrt{5})$, for the misfit parameter of the FK model, in contrast to the former value $M F=2 / 3$. Results for $M F=1 / 2$, the global minimums and

\subsection{Case $N=8, M F=1 / 2$}

The $M F=1 / 2$ is quite more problematic for some $N$ values than the former case of $2 / 3$. First we discuss the case $N=8$ which is actually not totally normal. To make the problems clearer, we will go deep into the details of the chain with a small number of atoms where we can study the PES. The NT to direction $(1,0, \ldots, 0,1)^{T}$ leads over an $\mathrm{SP}_{2}$ which, however, maybe not explicitly involved into a tilting in this direction.

Figure 6, left bottom panel, shows a 2-dimensional $\mathrm{PES}$ section over axes along the two negative eigenvectors of the $\mathrm{SP}_{2}$. A tilting along the direction of the NT will move together the starting minimum, below left, and the first $\mathrm{SP}_{1}$ on the right hand side. This pathway is enhanced by the tilting, compare the right panel. At the same time, the $\mathrm{SP}_{2}$ and the left $\mathrm{SP}_{1}$ move towards each other: the second path over the left $\mathrm{SP}_{1}$ will be inhibited. For the 'reaction path' of a tilting only counts the height of the 

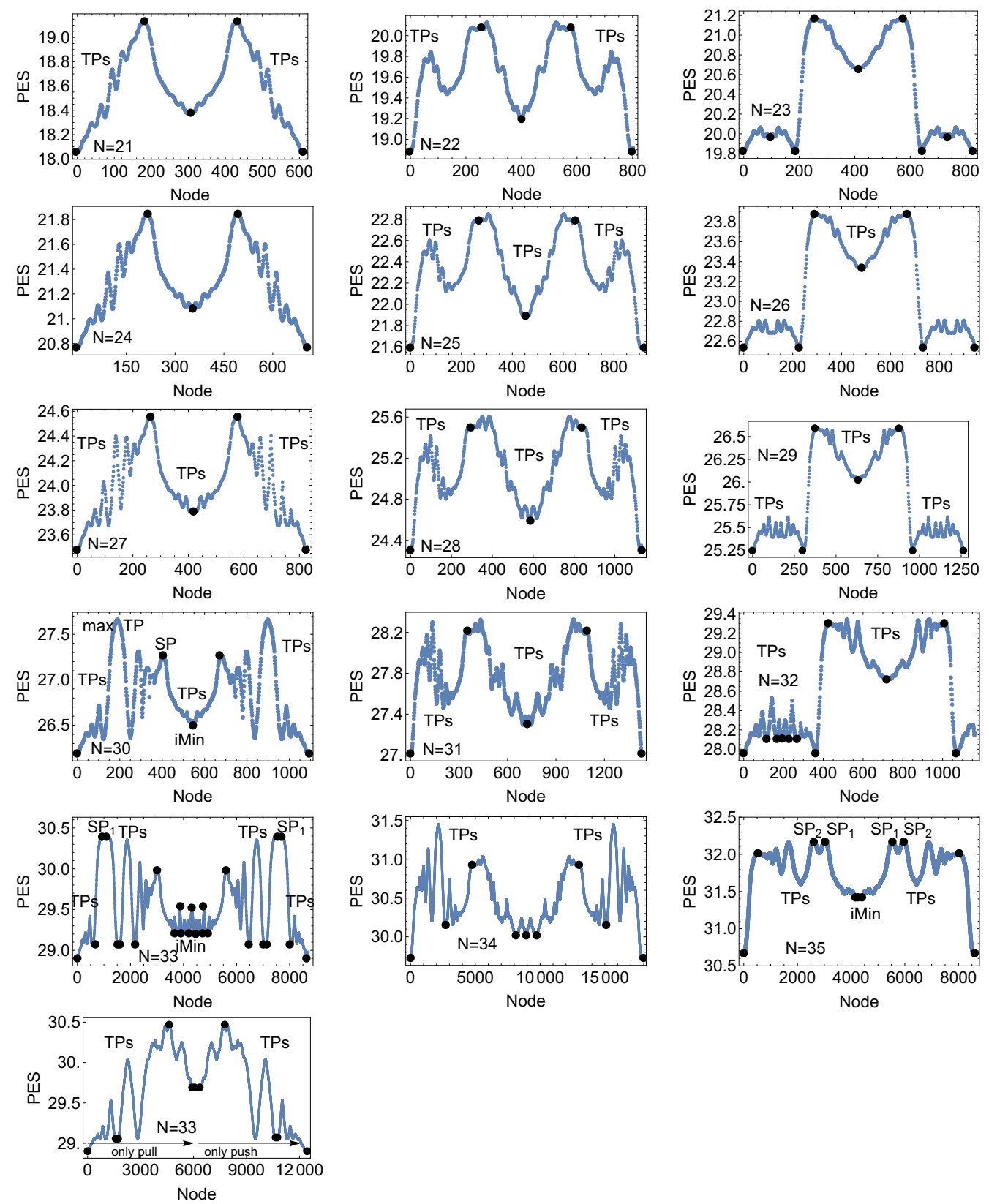

Fig. 4. Misfit parameter 2/3. Energy profiles over the NTs to direction $(1,0, \ldots, 0,1)^{T}$ (with the exception of the last panel). Black bullets depict stationary points of the PES. Note that for $N=25,28$, and extremely for $N=30$, and 34 , some TPs are higher than the SPs. The NTs for $N=30$ and 34 possibly cannot serve for a model of a successful tilting. In the case $N=33$ we calculated two different pathways, both are probably not the correct LEP. For $N=33$ to 35 we could find the NT after some shortenings of the predictor step length. Here many TPs emerge in the cape, and also in the inner well of the intermediate minimum. A further difficulty we find in the cases $N=33$ and 35 , see text.

corresponding right $\mathrm{SP}_{1}$. The $\mathrm{SP}_{2}$ is in every case circumvented. Thus, the NT which describes the inner parts of the FDSPs curve is not a model for a 'reaction path' here, at least not on the part which includes the $\mathrm{SP}_{2}$. In the left bottom panel of Figure 6 we have included further NTs to the axis-directions (thin black) for comparison, and a singular NT (red) which meets the valley ridge inflection point of the PES [25]. The set of points which fulfills the relation $\operatorname{det}(H)=0$ (green curves) describes the 'static frictional force' $[5]$ as the minimal force needed to initiate sliding for the corresponding crossing NT. Under the tilting, corresponding neighbouring stationary points can coalesce here, and the barrier of an $\mathrm{SP}_{1}$ can break down. In chemical papers, the force is named 'critical force', and the point on the PES is the barrier breakdown point. 

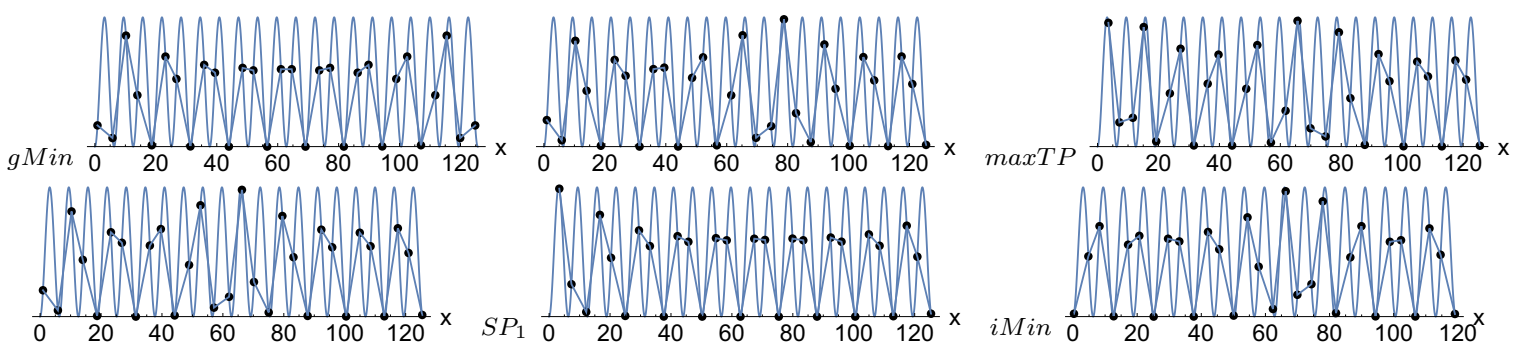

Fig. 5. $N=30$ and misfit $2 / 3$ (compare the panel $N=30$ in Figure 4 and Scheme 2). Top: schematic picture of the global minimum, the structures with minimal energy before the maxTP, and the maximal TP. Bottom: the structure with minimal energy after the maxTP, the $\mathrm{SP}_{1}$ and the iMin.
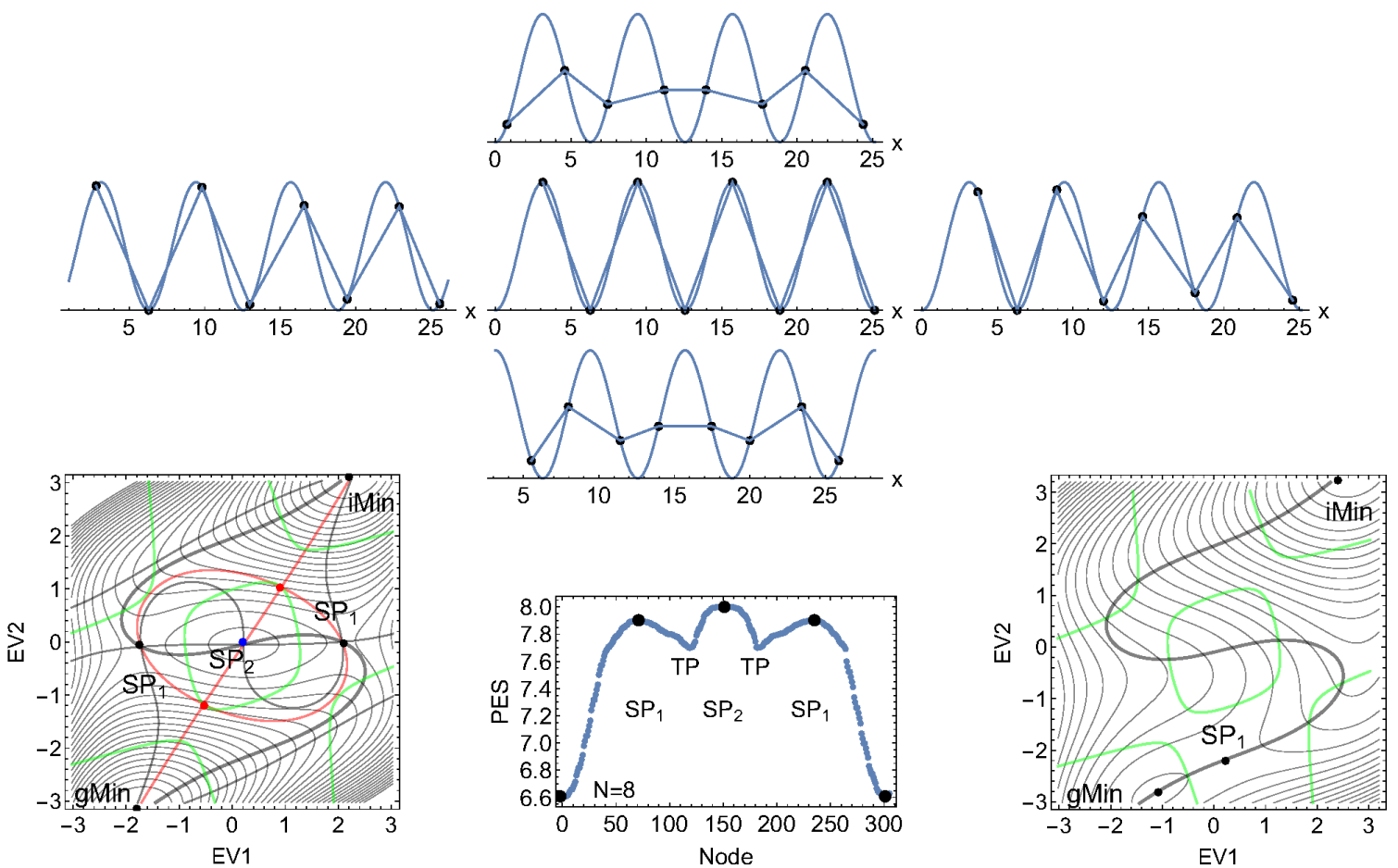

Fig. 6. Case $N=8$ for $M F=1 / 2$. Top three lines: Picture of the global minimum in the first line, the 'left' $\mathrm{SP}_{1}, \mathrm{the}^{\mathrm{S}} \mathrm{P}_{2}$, and the 'right' $\mathrm{SP}_{1}$ in the second line, and an intermediate minimum in the third line. The two minimums have the same energy. The connection over the MEP between the two minimums can go the left, or the right way. Bottom left: 2D PES section around the $\mathrm{SP}_{2}$. The NT (black) to direction $(1,1)^{T}$ turns up after passing the $\mathrm{SP}_{1}$ to the $\mathrm{SP}_{2}$ and then goes down to the other $\mathrm{SP}_{1}$, and to the final intermediate minimum on top. The red curve is a singular NT, compare Ref. [25], two thin black curves are the two NTs to the axes-directions, $(1,0)^{T}$ and $(0,1)^{T}$, and the green curves depict the $\operatorname{det}(H)=0$ set $[25]$. Bottom centre: energy profile over the NT to direction $(1,0, \ldots, 0,1)^{T}$. The SP at the top at node 150 is of index 2 . Bottom right: Tilted $\mathrm{V}_{F}$ with abstract tilting direction $-0.2(1,1)$ along the fat black NT. The former left $S P_{1}$ and the summit, $\mathrm{SP}_{2}$, are coalescented, and from the right $\mathrm{SP}_{1}$ only a small barrier remains. Usual thermic noise will move the chain from gMin at the bottom to the much deeper new iMin on top along the steepest descent, but not along the 'wavy' NT.

It is not always correct that the global minimum is unique as it is stated elsewhere [74]. We found here a special new property of the case $N=8$ with $\mathrm{a}_{0}=1 / 2 \mathrm{a}_{s}$. The chain has two different symmetric global minimums of the same energy, see Figure 6, first and third line. But the two different MEPs connecting the two minimums are both non-symmetric throughout.

\subsection{Further series to $M F=1 / 2$ with $N=10$,}

In this subsection we increase the number of particles for the misfit parameter $M F=1 / 2$. Some minimum structures, as well as the energy profiles over the NTs, are shown in Figures 7 and 8 . The minimum structures develop regularly, however, the NTs show more and more TPs on their ways. Beginning with $N=14, N$ even, an SP 

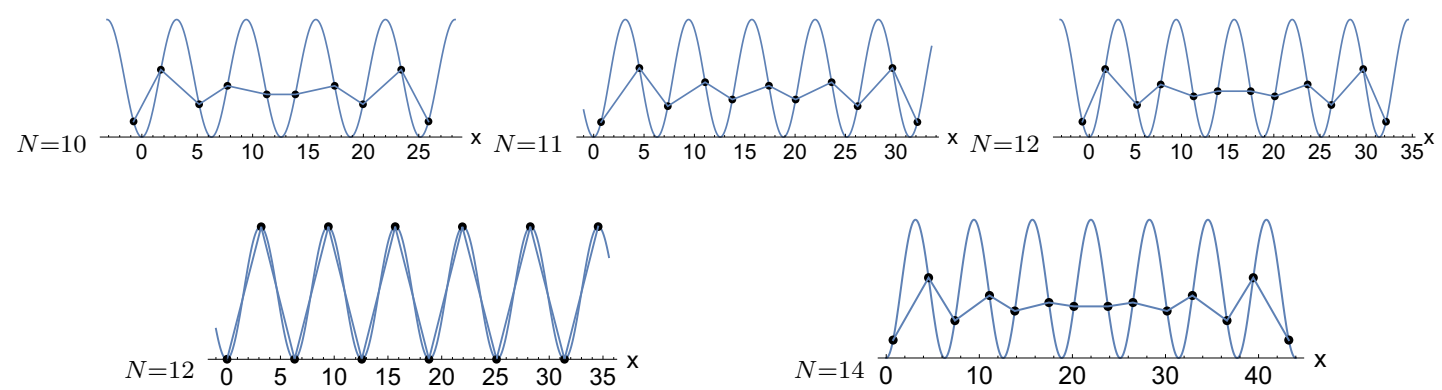

Fig. 7. Misfit parameter 1/2. Top line: three minimum chains for $\mathrm{N}=10,11,12$. Bottom left: structure of the labile $\mathrm{SP}_{2}$ of the 12-chain. An MEP will circumvent this point. Bottom right: minimum for $N=14$. The cases of even $N=10,12$, and 14 are quite similar. However, the case $N=11$ is asymmetric. There also emerges an SP of index 3 with a structure like the $\mathrm{SP}_{2}$ of the case $\mathrm{N}=12$.
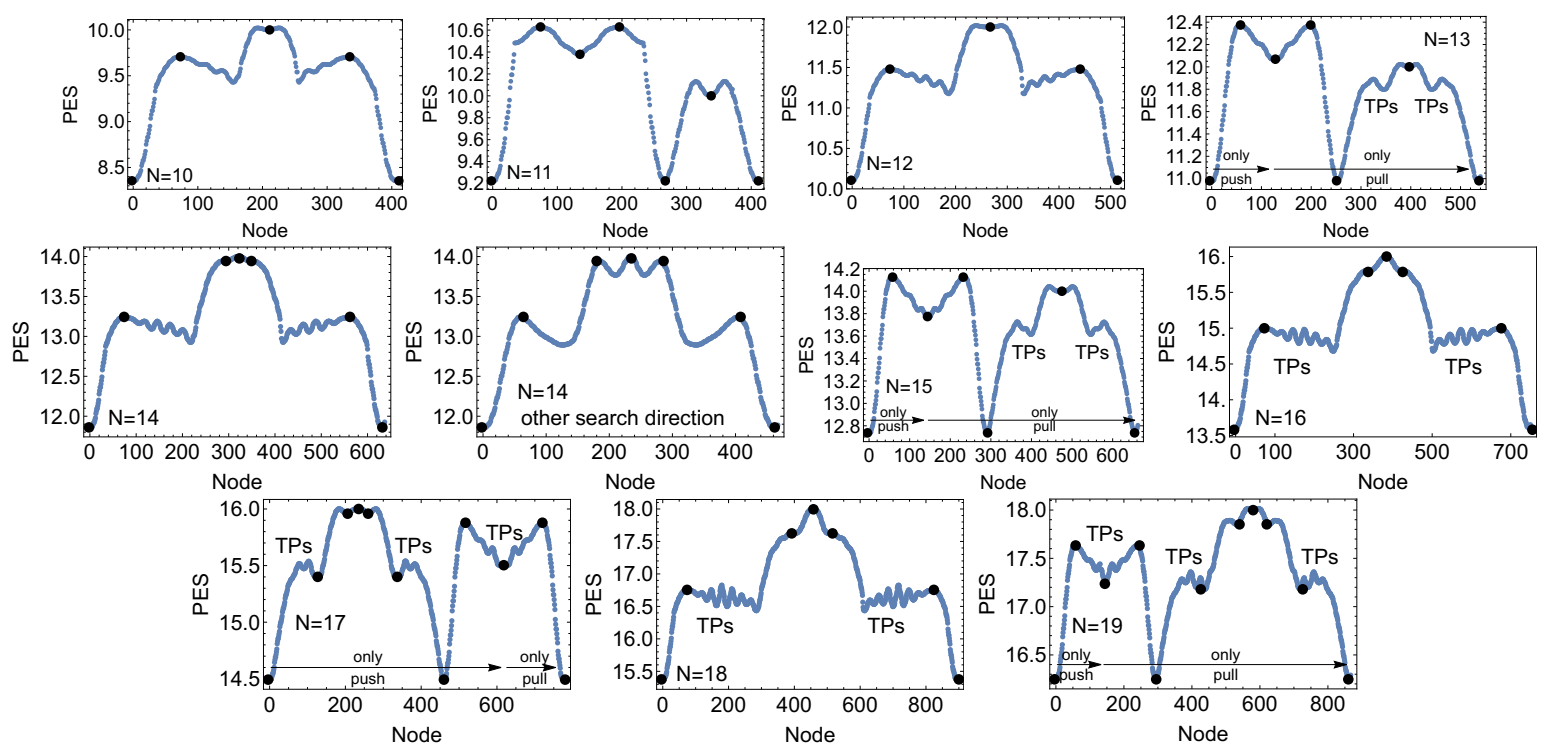

Fig. 8. Misfit parameter $1 / 2$. Energy profiles over the NTs for $N$ from 10 to 19 . NTs usually go to direction $(1,0, \ldots, 0,1)^{T}$, however, the second case $N=14$ is the NT to the SP-direction. But this NT also turns up to the $\mathrm{SP}_{2}$ and an $\mathrm{SP}_{3}$, and does not represent an MEP here, either. For $N=13,15,17$ and 19 we draw a combined profile for first an NT to push-only-, and second a part to pull-only direction, see text.

of index 3 on the NT additionally emerges here. Again, the 'inner' parts of the NT over these SPs of higher index are not the pathways of the sliding of the chain after a tilting. On such parts of the NT moves the other SP of index 1 which is not involved in the tilting.

The odd $N$ cases of misfit parameter $1 / 2$ all have an asymmetric global minimum. Then one has the corresponding mirror structure with the same energy. The profile on the transition from one minimum to its mirror is a kind of promontory for the global profile. However, these foot-hills increase with $N$, and for $N=17$ the profile over the NT for the 'promontory' becomes higher than the transition to the usual intermediate minimum for the 'breakthrough' through the PES mountains.

Beginning with case $N=13$ and $N$ odd we still find a strange, new situation: the NT to push- and pull direction is a singular NT which follows a direct pathway after the global minimum to an SP of index 2 with energy 13.7207 for $N=13$ (and then still to a peak over an SP of index 3 for $N=13,15$, and of index 4 for $N=17$ ). An example of a singular NT can be studied in Figure 6, bottom left panel, the red curve there. The singularity is characterized by a bifurcation point of the NT at a valley-ridge inflection (VRI) point of the PES. Starting at the minimum, the singular NT follows the minimum bowl up to the VRI and then it can follow straightforwardly the branch to the $\mathrm{SP}_{2}$ on a ridge. Of course, in the neighbourhood of the $\mathrm{SP}_{2}$ usually (at least two) SPs of index one exist. If the NT to push- and pull direction has found the $\mathrm{SP}_{2}$ then we assume that an NT to another direction will find a corresponding $\mathrm{SP}_{1}$.

To avoid the pathway of the singular NT to SPs of a higher index, we changed the search direction of the NT to find the $\mathrm{SP}_{1}$ for the low energy path through the PES. From the start we used the only push, $(1,0, \ldots 0)^{T}$, up to the intermediate, at node 128 for $N=13$, and from there the 

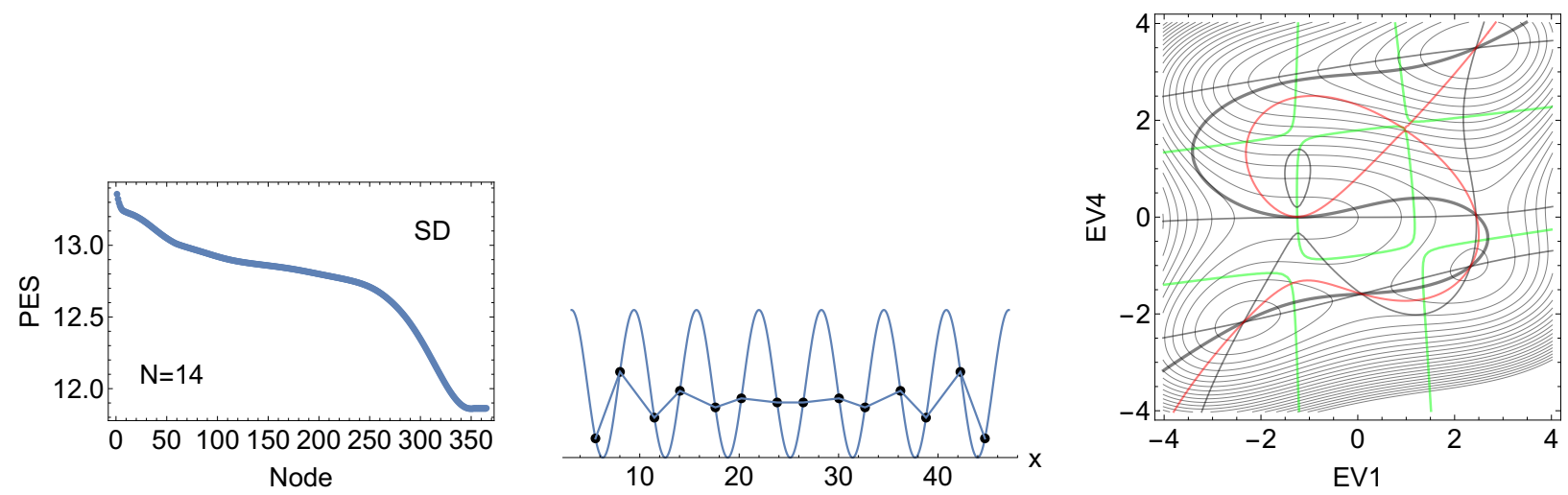

Fig. 9. Left: steepest descent for $N=14$, misfit parameter $1 / 2$, from $\mathrm{SP}_{1}$ to the 'next' minimum moved by $a_{s}$ along the axis. A 'direct' NT from the original minimum to the moved one, without the passage of the higher index SPs, could not be found. Center: Schematic picture of iMin for $N=14$, misfit 1/2, at the end of the SD from $\mathrm{SP}_{1}$. It has the same energy like the 'global' minimum. Right: 2D section around $\mathrm{SP}_{2}$ : the one extra iMin right below is artificial. Only the two other minimums lead to the former gMin or iMin.
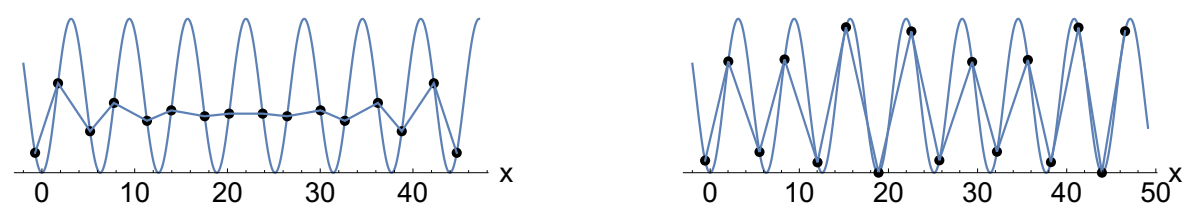

Fig. 10. Misfit parameter $1 / 2, N=16$, Schematic picture of the stationary points global minimum and an $\mathrm{SP}_{2}$. There also emerges an SP of index 3.

only-pull direction, $(0, \ldots, 0,1)^{T}$. The first direction leads from the intermediate minimum further, and 'false' up into the mountains to energies of 15 units, but the second, the pull-only direction, correctly finds the way out of the PES along the low energy path. Of course, for a tilting experiment one has to search a common direction for both parts of the pathway. So, $N=13$ may be the first critical value of the dimension, $N_{c r}$, here.

For $N=17$, the important SPs of index one for the low energy pathway are at nodes 517 and 720 , in the panel bottom left in Figure 8. The SPs of index one on the left hand side are on some 'pre-mountains'. The NT to push direction here additionally leads also over SPs of a higher index. This NT is followed up to the intermediate minimum at node 618 . The end of the pathway is the NT to the pull-only direction starting at the intermediate minimum. Because the 'pre-mountains' increase and increase for odd $N$, one can suppose that that it will cause more difficulties for a tilting of the chain.

The case $N=14$ is further discussed in Figure 9 . If we imagine the NT along the push- and pull direction to the $\mathrm{SP}_{1}$ then we have the pathway where the two stationary points, the global minimum and $\mathrm{SP}_{1}$, move towards each other and coalesce at least, for a height enough external force. Then the way to the next moved minimum, shown in the centre panel of Figure 9, is free. But this way is not represented by the former NT; only a steepest descent describes it. A coarse 2D projection of the relation is given by the right panel of the PES around the $\mathrm{SP}_{2}$.

Here for $N=14$, we additionally tested another search direction, the eigenvector direction along the $\mathrm{SP}_{1}$ valley.
The corresponding energy profile is shown in a central panel of Figure 8. The many small TPs of the former NT at the left panel, near the $\mathrm{SP}_{1}$, disappear, but near the central $\mathrm{SP}_{3}$ new TPs emerge. The global shape of the profile is, however, similar to the push- and pull direction. This NT also leads over the $\mathrm{SP}_{3}$.

The case $N=16$ is shortly discussed in Figure 10. In contrast to case $\mathrm{N}=8$, now the $\mathrm{SP}_{2}$ has a more complicated structure.

\subsection{Case $N=20, M F=1 / 2$}

We still discuss the 'abnormal' case $N=20, M F=1 / 2$. Some structures of this chain are shown in the first two rows of Figure 11 where one can imagine a movement of the chain along its axis through the different intermediates. In contrast, if we fixed the natural chain with spacing $a_{0}=a_{s} / 2$, what is numerically possible, and if we abstractly moved it over the on-site potential, it had to surmount a barrier of 10 energy units. It is exactly the value of $N / 2$. This is in strong contrast to the barrier of the case of misfit $2 / 3$ where usually barriers are less than or equal 2, shown elsewhere [26]. Here again the tilting along the push- and pull direction is possible up to the former $\mathrm{SP}_{1}$, but the relaxation must again be described by steepest descent, compare Figure 11, right panel at the bottom row. The NT describes the movement of the higher SPs together with its inner parts. It is not of interest for the tilting of the chain. 

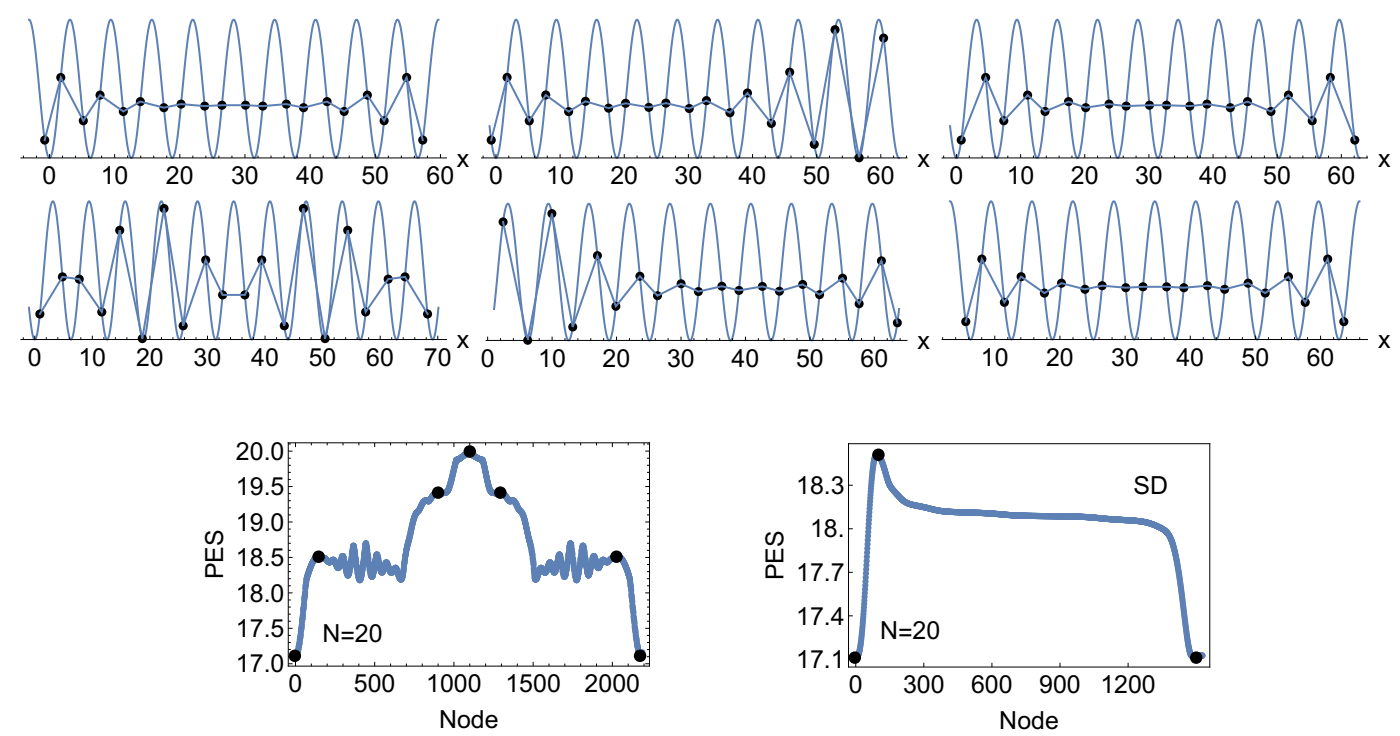

Fig. 11. Misfit parameter $1 / 2, N=20$. Top: schematic picture of the global minimum, the 'left' Sp and an iMin in the first line, and a high iMin, the 'right', mirror SP and the $2 \pi$ moved global minimum in the second line. Here again an $\mathrm{SP}_{3}$ emerges. Bottom left: energy profile over the NT to direction $(1,0, \ldots, 0,1)^{T}$. Black bullets depict stationary points of the PES. Here we did not find a deep valley with a global pathway through the mountains. The NT leads after the $\mathrm{SP}_{1}$ over a pass of the mountains with barrier 3 being also an SP of index 3. Right: energy profile over the two SD paths from $\mathrm{SP}_{1}$. The two parts are combined together. Left is the global minimum, right emerges an intermediate with equal height which is not met by the former global NT.

It seems that a critical $N_{c r} \approx 13$ to 20 for misfit $1 / 2$ will be somewhat before that one of the other parameter $2 / 3$ with $N_{c r} \approx 30$ described in the former Section.

\subsection{Comparison with the external force $F(1,1, \ldots, 1,1)^{T}$}

The global minimums for the misfit parameter $1 / 2$ are highly symmetric. An additional excitation with the highly symmetric force $F(1,1, \ldots, 1,1)^{T}[29,30,32]$ takes place along a singular NT in a symmetric subspace of the coordinates. This NT usually crosses the 'global' SP of the PES where half of the atoms are in the minimum well of the $(1-\cos x)$-function, but the other half is on top of the on-site potential. More exactly, if either $N$ is even then $N / 2$ atoms are on top, or if $N$ is odd then $(N+1) / 2$ atoms are on top. The energy of this SP is $2\left[\frac{N+1}{2}\right]$ and so the barriers are very higher than in Table 2 . And the barriers increase with $N$. Additionally, the 'global' $\mathrm{SP}$ is an SP of an index higher than one. Thus, the corresponding singular NT does not describe an LEP.

\section{Misfit reciprocal golden mean $M F=2 /(1+\sqrt{5})-$ series $N=6$ to 69}

We further use $v=k=1$ in equation (1). The 'structural mismatch', or the 'lattice mismatch', by the reciprocal golden mean [72] causes an non-periodic behaviour of the different chains to the tested length $N$. First we report on the profiles of NTs to the push- and pull direction for $N$ from 6 to 30 in Figure 12. All the NTs are obtained without numerical difficulties. Some 'mild' TPs emerge which are near the saddles on the NTs. Only the case $N=23$ has TPs which are a little higher than the $\mathrm{SP}_{1}$ which governs the barrier height. Figure 13 reports on the cases beginning with $N=31$. The first case with a rocky road for the NT is $N=36$. The profile is calculated with the usual push- and pull direction. Additionally, we had to sharpen the usual corrector threshold, which is in other dimensions $10^{-8}$, to the new value $5.5 * 10^{-12}$. The profile shows a further problem in higher dimensions. The three points of the intermediate minimum between nodes 500 and 750 are three times the same structure. The NT programme is turned back after a TP two times, but in the third try it finds the way to the outgoing $\mathrm{SP}_{1}$ and to the moved global minimum. Here we meet a numerical problem which is not relevant for an experimental tilting: The chain will slide through the LEP under a tilting, if such an LEP exists. If one has found the $\mathrm{SP}_{1}$ for the barrier to the intermediate minimum, and this is iMin itself, then it is very probable that the full path exists.

The peak of the red part of the shown profile in panel $N=36$ is not a TP of the NT, however, it is a numerical error where the NT also turns back its direction and goes back the former uphill way, now downhill. Such putative TPs are sometimes caused by the inaccuracy of the numerical programme. To detect such a case one has to control the movement of the chain along its axis. One can overcome the problem by shorter predictor steps, see some of the following panels where more and more numbers of nodes are used. (For the explanation of the problem, we used this not fully correct profile in the $N=36$ case.) 

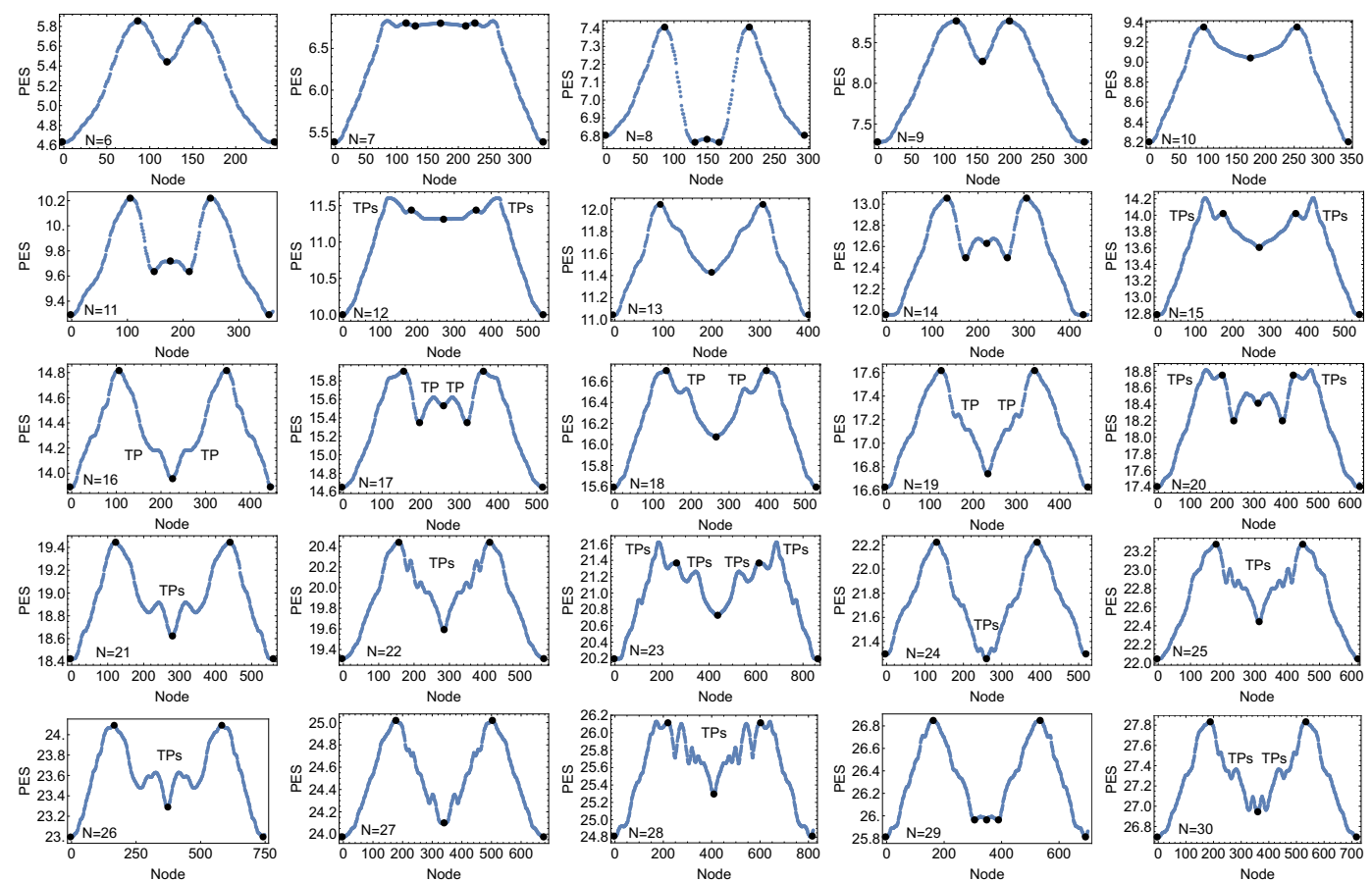

Fig. 12. Energy profile over NTs for the misfit parameter reciprocal golden mean. Black bullets are stationary points of the PES. The NTs are calculated to pull- and push direction $(1,0, \ldots, 0,1)^{T}$. Beginning with $N=12$, here 'mild' TPs emerge which are near the saddles on the NTs. For $N=23$ two TPs increase stronger over the $\mathrm{SP}_{1}$.

Some remarks, or comments are in order to special dimensions. First, the Fibonacci numbers 13, 21, 34, 55 for $N$ do not play any special role, in contrast to a statement of Ref. [75].

The case $N=44$ is the first for the reciprocal golden mean where we did not find a single NT along the LEP. We had to split the calculation into two parts like in some cases for $M F=1 / 2$. The profile for panel $N=44$ is a combined curve from the two NTs to two different directions. First up to the iMin region between node 350 and node 550 , we use the only-push direction, and from the last iMin-structure we use the only-pull direction. So we obtain a profile which may describe a nice LEP. But possibly, a tilting experiment with one of the used search directions may go wrong. We have to leave it open that here exists a unique NT to a common direction. From the point of the theory of the NTs, such a common direction does not need to exist! We only know that every NT connects stationary points; however which points is open, of course.

For the case $N=47$ we present two panels. The red profile belongs to an NT to the unique push- and pull direction starting in the intermediate minimum. The NT finds a usual $\mathrm{SP}_{1}$, however then it turns uphill to a higher $\mathrm{SP}_{2}$, and it then goes further to the different 'post'-SP $\mathrm{S}_{1}$. Here no further iMin is on the path. This path would be not a good device for a tilting because the second part after the $\mathrm{SP}_{2}$ can act as an inhibition of the movement of the chain. There the two different SPs can coalesce and so increase the barrier. But, nevertheless, here exists a LEP. We find it by an analogous trick like in case $N=44$. The blue profile of the next panel, also for $N=47$, is a combined curve from the two NTs to two different directions. First up to the iMin in the centre, we use the only push direction, and from the iMin we use the only-pull direction. So we obtain a profile which may describe an LEP through the 47-dimensional mountains of this chain. But possibly, a tilting experiment with one of the used search directions may go wrong.

For $N=49$ we again have to use two numerical steps for the NT to only-push- and only-pull direction, one up to the iMin, and one from there. The threshold for the corrector is set to $10^{-11}$.

For $N=50$ the threshold for the corrector is set to $10^{-11}$, and the predictor steps are strongly shortened. Besides the numerics, here again emerge quite high TPs which may disturb the tilting.

The next case, $N=51$, is also a 'good' case where a nice low energy path emerges. In all cases we could find the main $\mathrm{SP}_{1}$ for the barrier of a sliding.

The problems continue with $N=54$ and uphill: the NT to the push- and pull direction usually only finds the 'pre$\mathrm{SP}_{1}$, and then it goes 'wrong' uphill in the mountains.

The last case where the push- and pull direction gives the LEP is the case $N=64$. However, its calculation was very difficult, as one may see by the number of nodes which we needed. Single push or single pull NTs find here another intermediate minimum anywhere in the PES mountains.

In the cases $N=66,67$, and 69 we get three times nearly the same SP. Here the NT turns around, probably after the $\mathrm{SP}_{1}$ it passes an $\mathrm{SP}_{2}$ and then goes back to an equal $\mathrm{SP}_{1}$. 


\begin{tabular}{|c|ccc|}
\hline $\mathrm{N}$ & gMin & $\mathrm{SP}_{1}$ & barrier \\
\hline 6 & 3.8832 & 4.6331 & 0.750 \\
7 & 5.3799 & 6.8042 & 1.424 \\
8 & 6.8032 & 7.4083 & 0.605 \\
9 & 7.2797 & 8.7663 & 1.487 \\
10 & 8.2028 & 9.3504 & 1.148 \\
11 & 9.2923 & 10.2197 & 0.927 \\
12 & 10.0009 & 11.4389 & 1.438 \\
13 & 11.0443 & 12.0459 & 1.002 \\
14 & 11.956 & 13.0573 & 1.101 \\
15 & 12.7878 & 14.0209 & 1.233 \\
16 & 13.9565 & 14.8160 & 0.925 \\
17 & 14.6509 & 15.9034 & 1.253 \\
18 & 15.5944 & 16.7041 & 1.110 \\
19 & 16.6279 & 17.6168 & 0.989 \\
20 & 17.4038 & 18.753 & 1.339 \\
21 & 18.4242 & 19.4457 & 1.022 \\
22 & 19.3119 & 20.4393 & 1.127 \\
23 & 20.196 & 21.3674 & 1.171 \\
24 & 21.2576 & 22.2225 & 0.965 \\
25 & 22.0468 & 23.2731 & 1.226 \\
26 & 22.9971 & 24.0926 & 1.095 \\
27 & 23.9753 & 25.0196 & 1.044 \\
\hline
\end{tabular}

\begin{tabular}{|c|ccc|}
\hline $\mathrm{N}$ & gMin & $\mathrm{SP}_{1}$ & barrier \\
\hline 28 & 24.8127 & 26.1138 & 1.301 \\
29 & 25.813 & 26.848 & 1.035 \\
30 & 26.6967 & 27.8337 & 1.137 \\
31 & 27.6048 & 28.7486 & 1.144 \\
32 & 28.6408 & 29.6278 & 0.987 \\
33 & 29.4465 & 30.6591 & $1.213^{*}$ \\
34 & 30.4031 & 31.4908 & 1.088 \\
35 & 31.3553 & 32.4238 & 1.068 \\
36 & 32.2131 & 33.4906 & $1.278^{*}$ \\
37 & 33.2117 & 34.2534 & 1.042 \\
38 & 34.0945 & 35.2334 & 1.139 \\
39 & 35.0087 & 36.3278 & $1.319^{*}$ \\
40 & 36.0146 & 37.0334 & 1.019 \\
41 & 36.8529 & 38.0517 & 1.199 \\
42 & 37.8065 & 38.8914 & 1.085 \\
43 & 38.7465 & 39.8285 & 1.082 \\
44 & 39.6182 & 40.8776 & $1.260^{*}$ \\
45 & 40.6126 & 41.6584 & 1.046 \\
46 & 41.4924 & 42.6359 & 1.144 \\
47 & 42.4180 & 43.5391 & $1.121^{*}$ \\
48 & 43.4007 & 44.4387 & 1.038 \\
49 & 44.2527 & 45.45 & $1.197^{*}$ \\
\hline
\end{tabular}

\begin{tabular}{|c|ccc|}
\hline $\mathrm{N}$ & gMin & $\mathrm{SP}_{1}$ & barrier \\
\hline 50 & 45.2149 & 46.2940 & $1.079^{*}$ \\
51 & 46.1418 & 47.2337 & 1.092 \\
52 & 47.0269 & 48.2705 & 1.244 \\
53 & 48.0158 & 49.0632 & 1.047 \\
54 & 48.8921 & 50.0416 & $1.150^{*}$ \\
55 & 49.8206 & 50.9372 & 1.117 \\
56 & 50.7889 & 51.8448 & 1.056 \\
57 & 51.6572 & 52.8504 & 1.193 \\
58 & 52.6154 & 53.6243 & $1.008^{*}$ \\
59 & 53.5372 & 54.6408 & $1.103^{*}$ \\
60 & 54.4333 & 55.5855 & $1.152^{*}$ \\
61 & 55.4200 & 56.4701 & $1.050^{*}$ \\
62 & 56.2949 & 57.4450 & $1.150^{*}$ \\
63 & 57.2250 & 58.3443 & $1.119^{*}$ \\
64 & 58.1858 & 59.2488 & 1.063 \\
65 & 59.0616 & 60.2314 & $1.170^{*}$ \\
66 & 60.1032 & 61.0599 & $0.957^{*}$ \\
67 & 60.9369 & 62.1532 & $1.216^{*}$ \\
68 & 61.8335 & 62.9810 & $1.148^{*}$ \\
69 & 62.8227 & 63.8740 & $1.051^{*}$ \\
.. & & & \\
101 & 92.4273 & 93.6346 & $1.207^{*}$ \\
\hline
\end{tabular}

Table 3. Energies of the $N$-chains for misfit parameter 0.618034 at stationary points, and the barrier. The $*$ means that no unique NT could be found for the LEP, thus one NT to only one direction. In contrast for every half way we used either only-pull or only-push direction. Note that the reported barriers can be somewhat over the lowest possible value if there exists another MEP, compare the case $N=23$ of $M F=2 / 3$ in Ref.[26]. If we found 'any' LEP, we stopped the calculation.

Such a behaviour is allowed by the NT theory. Compare Figure 6, panel left below for an 8-dimensional chain.

We have worked out, after a jump in $N$, the 'overwhelming large' case $N=101$. As one can see in the last line of Figure 13, the case is similar to the last former dimensions. Here are again many small peaks with TPs on the two NTs, but we meet a usual barrier, and the TPs are not too high. The case speaks for our feeling that the LEP will exist also for further dimensions. However, the complexity of the behaviour of the NTs with the many small extra peaks with their many 'useless' TPs will explode with increasing $N$.

\section{No Misfit between $a_{s}$ and $a_{0}$}

For comparison we still treat the case that $\mathrm{a}_{s}=a_{0}=2 \pi$, thus the MF parameter is 1 . We study the 'most commensurate' case [76,77]. It is quasi trivial, on a first sight, because every atom is in one well of the on-site potential, $x_{i}=(i-1) * 2 \pi$ for the global minimum, gMin. The FK potential energy is $V\left(\mathbf{x}_{g \text { Min }}\right)=0$ for all $N$. The general gradient vector is $g_{i}(\mathbf{x})=\partial V(\mathbf{x}) / \partial x_{i}=v \sin \left(x_{i}\right)+k\left(-x_{i+1}+\right.$ $\left.2 x_{i}-x_{i-1}\right)$ for $i=2, \ldots, N-1$ and $g_{1}(\mathbf{x})=v \sin \left(x_{1}\right)-$ $k\left(x_{2}-x_{1}-a_{0}\right), g_{N}(\mathbf{x})=v \sin \left(x_{N}\right)+k\left(x_{N}-x_{N-1}-a_{0}\right)$. Finally, the general Hessian matrix is $H_{i, j}=\partial^{2} V(\mathbf{x}) / \partial x_{i}^{2}$ where $H_{i, i-1}=H_{i, i+1}=-k$ and $H_{i, i}=v \cos \left(x_{i}\right)+k(2-$ $\left.\delta_{i, 1}-\delta_{i, N}\right)$ for $i=1, \ldots, N$. The gradient at gMin is equal to the zero vector, and the Hessian is positive definite in gMin. Further we continue to use $v=k=1$.

However, for a movement of the chain along its axis, the 'trivial' structure of gMin becomes a problem. Here the structures for a really low energy path do not exist. First we treat the pull- and push direction for an external force. Because of the high internal symmetry of the chain, in relation to the on-site potential, we get a singular NT in a symmetry subspace which leads over SPs of higher index, see the left panel of Figure 14. The involved structures of the 10-chain are depicted in Figure 15. Usually, besides an $\mathrm{SP}_{2}$ emerge lower SPs of index one. So, it is clear that the pull- and push direction is not a good choice for a LEP for an external force.

The second possibility is a half-NT to push-only direction, and from a reached (symmetric) intermediate minimum a start with a pull-only NT. The combined profile is shown in the right panel of Figure14. The pathway is lower in energy than the NT to push- and pull direction in the left panel, however, it is still not a path with such low energy like in the cases of the other misfit parameters in the former Sections. Because of the full regularity of the global minimum of the chain, it needs quite more energy to move a kink, or an anti-kink through the PES. The reached stationary points of the 10-chain on the combined NT are shown in Figure 16. The barrier for a movement of the chain is 7.894. It is an exorbitant value in comparison to chains with real misfit relations, in the former Sections. The high effort for the NT may come from the property of the chain that already the ground state has a kind of 'standard traffic jam' for its single atoms [28]. There is no simple possibility to move one atom into a next gap. Because here are no gaps. Here we cannot create a critical $N_{c r}$. The small dimensions are already critical. 

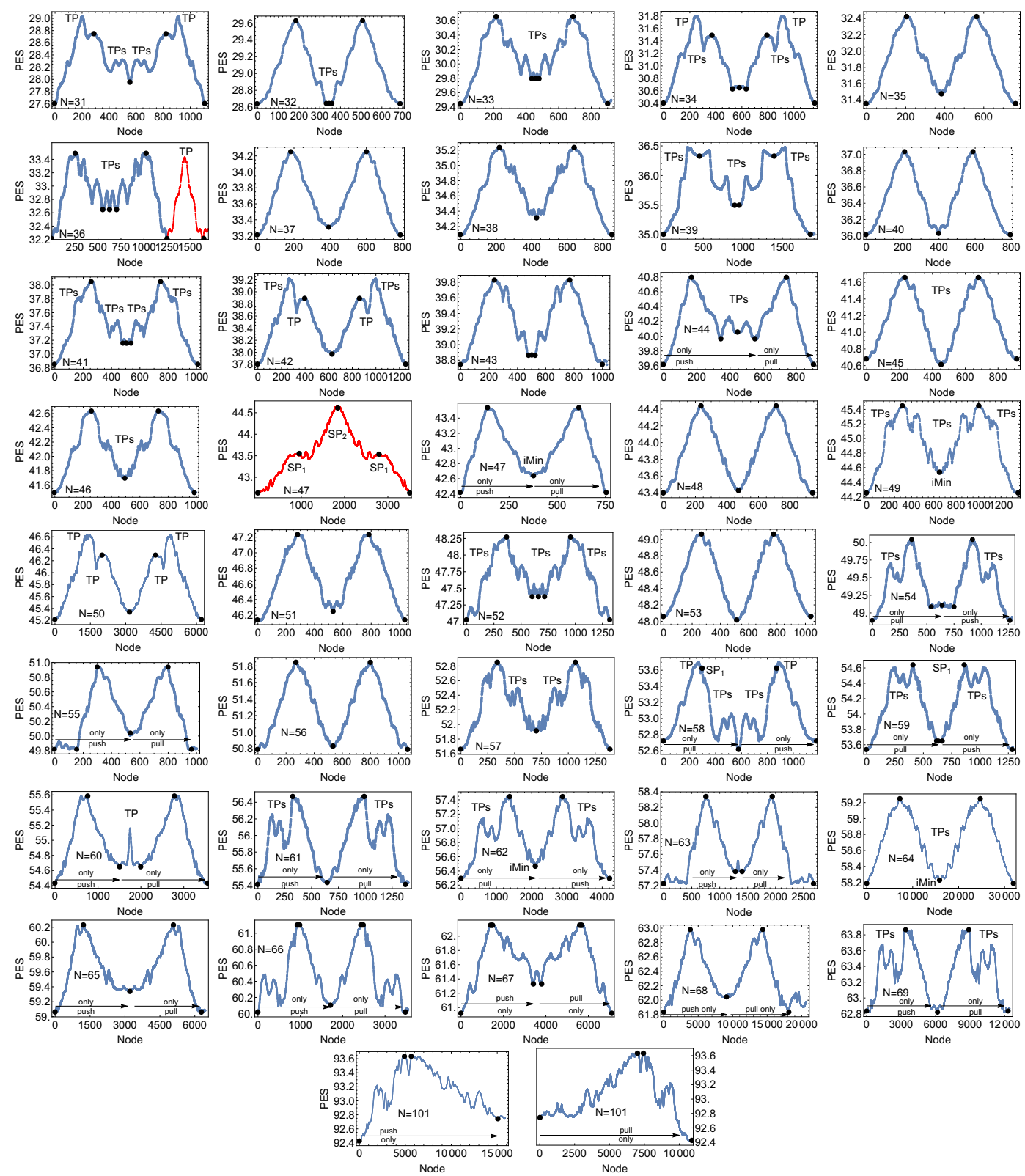

Node

Fig. 13. Energy profile over NTs for the misfit parameter reciprocal golden mean. Black bullets are stationary points of the PES. Peaks without a bullet are TPs. The NTs in the lower dimensional cases are calculated to pull- and push direction $(1,0, \ldots, 0,1)^{T}$. 'Mild' TPs emerge again. For $N=31,39$, and 50, two TPs increase stronger over the $\mathrm{SP}_{1}$. Red profiles (for $N=36$ in part, and $N=47$ ) describe degenerated NTs, see text. Profiles for the higher dimensional cases are obtained by two different NTs to pull- or to push direction only. See the arrows in the corresponding panels. Note the nice symmetry of the profiles even though different directions are used.

The kind of flat intermediate minimums like in the central panels of Figure 16 come in for the first time in dimension $N=8$. Before that, up to $N=7$, 'only' an $\mathrm{SP}_{1}$ forms the barrier on the MEP for a full movement of the chain. Compare the first seven atoms in the first panel top left of Figure 16. However, the push- and pull direction for an NT is too symmetric. The corresponding NT is singular. It leads over a 'global' SP of higher index where all atoms are possibly on top of the on-site potential, like in the central panel of Figure 15. There for $N=10$, the SP has index 4 . The energy is 20 , and in general case the energy of this 'global' SP is simply $2 N$. For the 'global' SP we guess that

$$
\text { if } \mathrm{N} \equiv\left\{\begin{array}{l}
1 \\
2 \\
0
\end{array}\right\} \bmod 3 \text { the index is }\left\{\begin{array}{c}
{[N / 3]+1} \\
{[N / 3]+1} \\
N / 3+1 / 2
\end{array}\right\}
$$

where $1 / 2$ means that here is an additional zero eigenvalue in the list, the point is a shoulder in the corresponding 

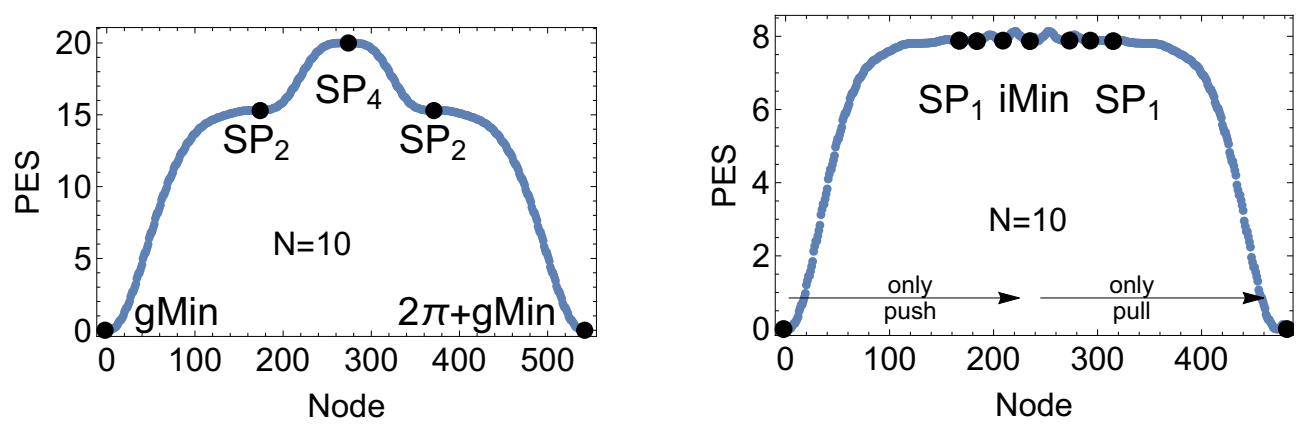

Fig. 14. Energy profiles for NTs for chain length $N=10$ and the $M F=1$. Left panel to push- and pull direction, right panel to a combined use of first push only, and second only-pull direction. The row of stationary points on top of the right profile consists of alternating flat $\mathrm{SP}_{1}$ and iMin, compare Figure 16.

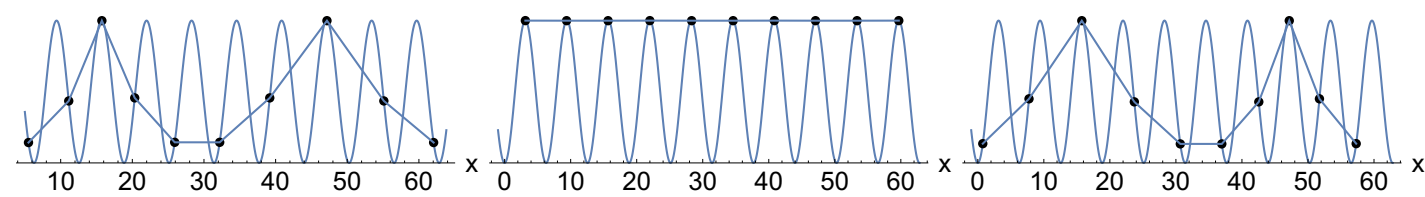

Fig. 15. Structures of the 10 -chain with $M F=1$ on the NT to push- and pull direction. The left panel is an $\mathrm{SP}_{2}$, the centre is an $\mathrm{SP}_{4}$, and the right panel is again an $\mathrm{SP}_{2}$, the mirror image of the left one. The $\mathrm{SP}_{4}$ is here the 'global' $\mathrm{SP}$ for $M F=1$. All atoms are on top of the sinusoidal function.
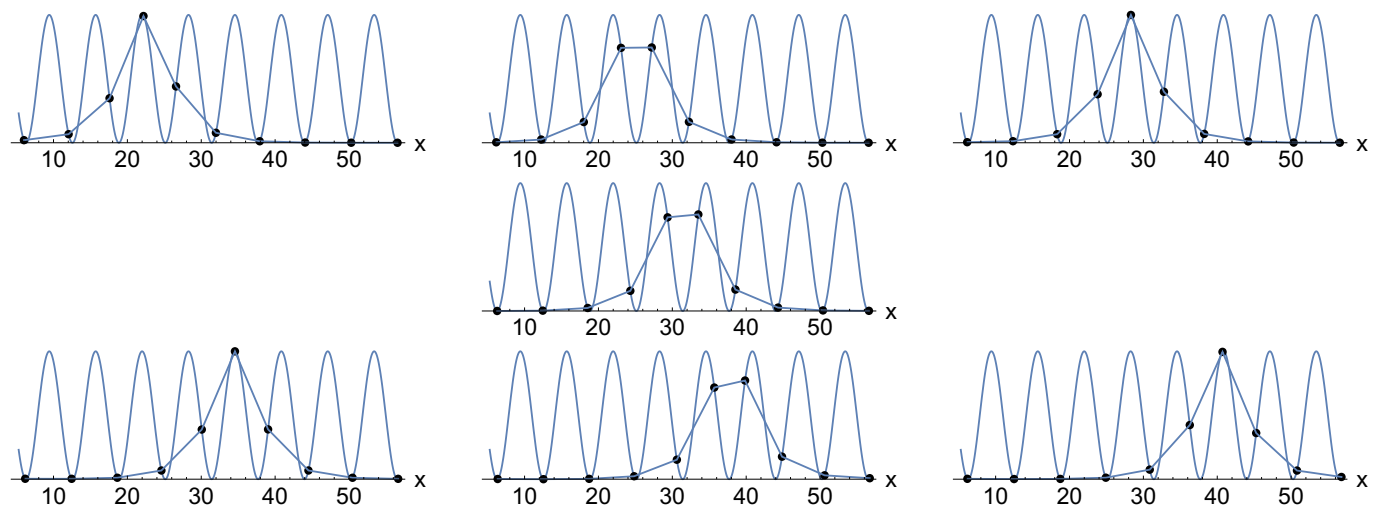

Fig. 16. Structures of the 10 -chain with $M F=1$ on the combined NT to push- or pull direction. The panels alternate the $4 \mathrm{SP}_{1}$ and the 3 iMin structures. The latter depicts an anti-kink which moves through the chain.

direction. Such SPs of an higher index should be avoided by the moving chain.

To explore the MEP, one has again to use two different search directions. It works for only-push direction up to the $\mathrm{SP}_{1}$, and only-pull direction downhill to the next global minimum. The barriers are 7.647 for $N=5,7.778$ for $N=6,7.858$ for $N=7,7.873$ for $N=8$, and so on. In the last case, as it was already reported, the barrier is formed by quasi a line of alternating $\mathrm{SP}_{1}$ and iMin. This pattern seems to continue for all $N$.

In Figure 17 the energy profile for two combined NTs in the case $N=20$ is shown. Like on a straight, flat line, the consecutive stationary points are ordered and form the barrier: $N-6=14 \mathrm{SP}_{1}$ and $N-7=13$ intermediate minimums, iMin. Their energy differs only minimally. The Peierls-Nabarro barriers on the flat pathway from the first pre-SP ${ }_{1}$ to the last post-SP ${ }_{1}$ are near zero. The consecu- tive $\mathrm{SP}_{1}$ and iMin change between 7.892 and 7.869 energy units. The barriers are 0.023 units. The stationary points of this flat way describe the movement of an anti-kink through the chain under the corresponding external force. However, the NT deviates more and more from the flat MEP between the stationary points. Compare Figure 14 right panel, and Figure 17. The external force pushes the chain at the first atom. So, the inner atoms represent 'other' directions. The NT to push only consists of more and more peaks with high TPs. Here for $N=20$, the TPs reach 16 energy units. At least, on the last arc before the central iMin, the NT 'escapes' over two further high stationary points, an $\mathrm{SP}_{2}$ and an $\mathrm{SP}_{1}$, both are near neighbours, but both are far away from the structure of the $\mathrm{SP}_{1}$ before and the central iMin next. All these arcs are roundabout ways, and their energy increases to twice the value of the barrier, but of course the energy is less than 


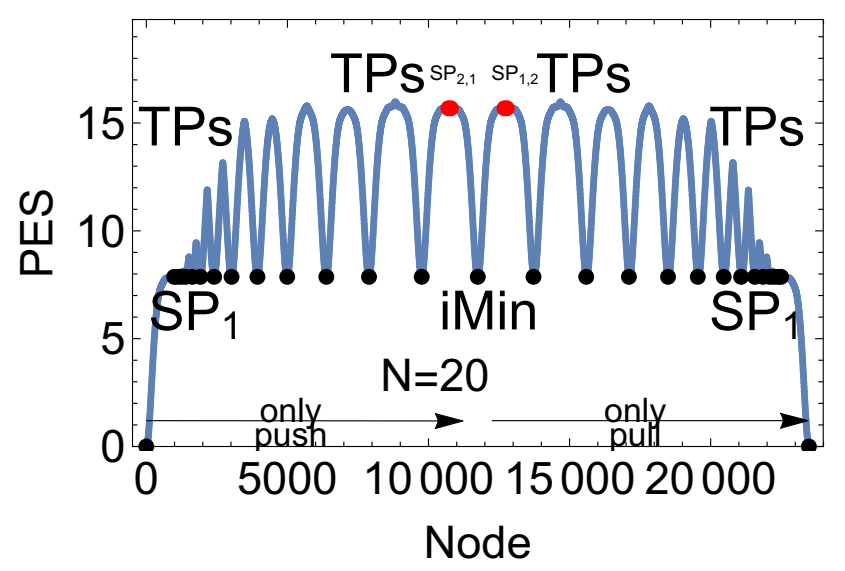

Fig. 17. Energy profile for the 20-chain with $M F=1$ on the combined NT to only-push- or only-pull direction. The alternating $\mathrm{SP}_{1}$ and iMin structures form the barrier. They are the lower spikes of the NTs. They describe an anti-kink which moves through the chain on a really equal level, here at maximally 7.892. However, the NTs which explore the stationary points do not lead along the valley floor, they do not directly connect the consecutive saddle-sink sequence, but they escape to much higher energies with TPs at the top of the peaks, and additional SPs of index 2 and 1 (red bullets). The MEP itself leads over all the black bullets.

the 40 units of the fully symmetric central SP of index 7 here, where $x_{i}=(2 i-1) \pi, i=1, \ldots, 20$. The barrier of the MEP of the moving anti-kink through the chain is on a nearly equal level 7.9. However, the problem that a single direction for the push of the long chain is not really well adapted, cannot be avoided. We get the unnecessary, large peaks of the NTs.

Anyhow, the NT to only push goes up to the central iMin. If we follow it further, then it turns up, and it crosses anywhere other SPs of higher energy and higher index. So, the stationary points of the MEP are well described by the combined two NTs to first push direction only, and second pull direction only. One may wonder that the pulland push direction itself does not lead along the path.

It is allowed to speculate that for an external force in only-push direction, since the amount is large enough to surmount the barrier breakdown point $[25,26]$ between the global minimum and the first pre-SP $\mathrm{SP}_{1}$, at an energy of 7.674 with a gradient norm of 0.517 , being the static frictional force, $F_{c}$, then the anti-kink may start to slide, and slide further along the MEP through the PES. The flat barrier looks like an invitation to superlubricity. However, the speculation will not be correct. Note that the NT describes the movement of the diverse stationary points on the PES under the external force. The way along the profile of the course of the corresponding NT describes the energy to surmount the current next $\mathrm{SP}_{1}$. This will increase the value near 16 energy units. And it holds only for the first half of the barrier, up to the central iMin. Then the only-push NT still more deviates from the flat barrier. The full pathway from the left global minimum at zero energy to the right global minimum at zero energy is only one step of the movement of the chain along its axis. A sliding would need many such steps. It is so complicated because the structures of the chain along the LEP are no uniform sliding, in contrast to an assumption of Ref. [44].
No, the anti-kink which moves through the chain changes continuously the inner structure of the chain.

If $N$ increases to larger and larger values, and at least to infinity, one could 'forget' the first step of an exitation from the global minimum of the chain to the pre-SP 1 at ' $N=-\infty+4$ ', for the first anti-kink. Then anywhere on the flat barrier one could move the anti-kink along the chain without a Peierls-Nabarro barrier, and this may emerge like a 'ground state' of the chain. The movement could emerge like a superlubrical sliding.

Another speculation may concern a dynamical behaviour of the atoms of the chain after a single large external push to the first atom, $x_{1}$. Here may emerge the first anti-kink in the first flat iMin in the well 4 of the on-site potential. How can the induced anti-kink wander by the impulse through the chain, if the first atom is shifted over the flat barrier with all the $\mathrm{SP}_{1}$ and iMin? Such a moving dislocation would not need to go along the peaks of the NT to pull-only-direction to atom $x_{1}$. It could go like a wave along the flat barrier. We leave open the question for further studies, see for example also the former Refs. [72, $76,78,79]$.

\section{Comparison}

We compare the three cases of the true misfit parameter. For the stationary states no trend can be detected; also for the 'most' incommensurate ratio of the reciprocal golden mean the barrier for a sliding does not disappear for higher $N$, either. But all barriers are less than 2, in contrast to what was approximated in an older work [42]. In Figure 18 we compare the different barriers of any obtained low energy path for the three misfit parameters and the different chain length. The slight reduction of the barrier for the different misfit parameters comes from the fact that different particles experience more opposite forces of the on-site potential. If one atom has to overcome a peak then another 
neighbouring atom slides down the next slope of its well. This property, however, depends on the overall density of the particles of the chain in the on-site potential. Note: the lattice forces never compensate totally, in contrast to an assumption in Ref. [75]. The Fibonacci numbers 13, 21, 34,55 for $N$, with exception of 8 , do not play any special role for the red points, in contrast to a statement of Ref. [75]. Clear differences between the misfit parameters

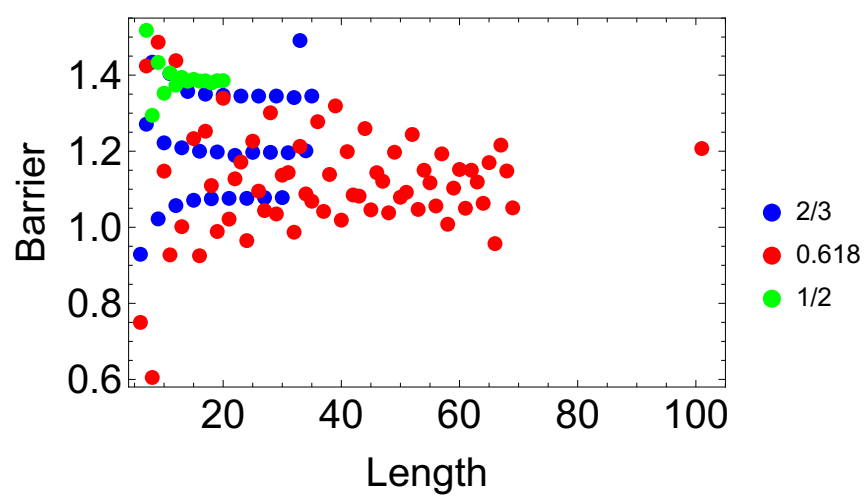

Fig. 18. Barriers of the calculated NTs for different chain length and the misfit parameters $1 / 2$, reciprocal golden mean, and $2 / 3$. For no misfit, $M F=1$, we find an equal barrier at $\approx 7.9$, which we assume to hold for all $N$.

emerge if we try to test a maximally possible chain length for useful NTs for a tilting of the FK model. Reasons are discussed in former Sections: large arcs of the NTs describing roundabout ways with high lying TPs, or the NT of interest becomes a singular NT and leads to SPs of higher index, on the PES quite over the searched LEP.

\section{Discussion of the low energy paths}

In the 'good' case when the found NT well adapts the LEP we will only have little and small extra peaks with TPs. Say, we treat the case $N=48$ of $M F$ reciprocal golden mean, compare Figure 13. Then we can assume that the NT follows the LEP near its valley ground. The chain changes along the LEP in a characteristic way. Say, we start with a pull only of the external force. Then at the start we cause an anti-kink under the first atoms of the chain, a compression. By the spring forces between the atoms, the anti-kink will move through the chain, but it needs more and more energy, the more atoms it involves. This continues up to the 'pre-SP'. From there the chain relaxes to the intermediate minimum, iMin, which may be now a compressed equilibrium. But usually it has a higher energy in comparison to the global minimum. The other half of the LEP may be adapted by a pull-only NT. Here we start at the iMin, but pull with the external force at the end of the chain. This causes a kink under the last atoms of the chain, a stretching of the distances of the outer atoms. The atoms have a rarefaction. Again, the kink may move through the chain, but it needs somewhat more energy, if more atoms are involved. With enough energy it will reach the post-SP, and from there the kink from the end, and the anti-kink of the compressed structure can unite and form the new global minimum moved by $a_{s}$.

The sliding of the chain over the substrate does not take place in fixed form. It goes on by a kind of wave of an anti-kink, or a kink along the chain, a moving dislocation. This is also the case for no misfit between the onsite potential and the springs of the chain, $M F=1$. But because here all wells of the on-site potential are equally filled by atoms of the chain, in the global minimum of the chain, one cannot exchange different fillings of atoms in the wells of the on-site potential like in the former cases. Any external force must first produce an anti-kink, or a kink, at the ends of the chain. This causes a larger effort than for true misfit parameters. However, here we then can move the produced dislocation with an equal energy height through the chain. We get an MEP of a very nice flat kind over a series of alternating $\mathrm{SP}_{1}$ and iMin from one end of the chain to the other. And this seems independent of the length of the chain. For our choice of parameters $v=k=1$, we have an equal height of the flat barrier of $\approx 7.9$ energy units.

\section{Conclusion}

We do not discuss any kind of kinetic friction here, because we only treat static properties of the PES of the FK model. Our calculations have been mainly developed in the tens-of-atoms regime. This falls considerably short of the scales at which one would like to understand energy trends and structural properties in materials. Recently, experiments are done with laser-cooled and trapped ions for insights into friction processes $[38,47,68,80]$. The systems try to emulate the FK model (1) for a small number $N$, where the chain of the interesting particles slides under an external force over the fixed rigid on-site potential. The FK model (1) is sufficiently complex for tests of basic concepts [31]. An only-pull force, for example, is an excitation by a localized point-like contact like in an atomic force microscope, or in a friction force microscope. However, we do here not study the dynamical behaviour of the chain under a push, a pull, or a vibrating external force. But it seems to be clear that in the cases with $M F \neq 1$, where two SPs and an intermediate minimum govern the low energy pathways, we will not get that an (anti)kink can propagate freely. This may be possible for the case $M F=1$, compare Figs. 14 to 17, where after an excitation of the chain over the first pre-SP we have a quasi flat path over all the next iMin and $\mathrm{SP}_{1}$. Note that any kind of dampings are also not treated here.

We again demonstrate in this paper that the stationary states like minimums and SPs of any index of the PES of the finite FK model (1) can be obtained by NTs (or any other minimization method [39]). It is not problematic if 
an equilibrium state is asymmetric, and the method is independent of the relation of the parts of the misfit parameter, the periodicities, $a_{s}$ and $a_{0}$. May they be rational or irrational, commensurable or incommensurable. The 'most irrational' case of the reciprocal golden mean may have some slightly lower MEPs, however, qualitatively, the case is the same like for rational misfit parameters, at least for small $N$.

The PES (1) is directly given in the FK model. We can execute all calculations of NTs which determine the diverse properties of the model. The accuracy can be high. For comparison and confirmation we controlled the results of NT calculations by usual minimization procedures of the Mathematica programme because the PES of the FK model (1) is a usual hypersurface where the known minimization procedures work. All NTs over the PES of FK chains are calculated numerically. Maybe some problems which we suffer in some cases come from the procedure for the following of the NTs. - But we are not 'indignant' by the necessity of numerical simulations. It is our only possibility to see the low energy pathways through the high energy PES of an FK chain. So we can present convincing numerical data.

NTs are especially appropriate to the driven FK model by a tilting force. By the calculation of a barrier breakdown point (BBP) on the NT of the driving force direction we can get the depinning transition $[25,26]$ and the gradient there is the critical force, the static frictional force. The critical phenomenon is reducible to a saddle-node transition [74], or a shoulder on the effective PES. All that are static properties of the effective PES.

NTs allow us to drive they in very different directions over the PES. The standard direction $(1, \ldots, 1)^{T}$ is physically well understandable. Our used directions in the higher dimensional cases, beginning with $N=6$, usually have to deviate from the standard direction. The corresponding valleys on the corrugated PES, which may be also very curvilinear, are better to follow by NTs which are adapted to the situation. So we work in the general case with the push- and pull direction, and in more complicated cases with a tilting which concerns only one end of the chain. However, so the individual peaks and valleys of the PES open, and so better adapted forces are to get; and may be experimental workers can construct an experiment where such better forces are applied to the chain, and with a lower load than it is necessary for a full movement of the chain in standard direction. We hope that this paper will stimulate further research in this vibrant research area.

The central finding of the paper is the existence of the LEP with a barrier of less than 2 energy units through the PES of all examples of true misfit parameters which are calculated. For the case of $M F=1$ we have a 'medium' path with a barrier of 7.9 energy units through the PES. Many LEPs are well adapted by the corresponding NT of the 'numeric' proof, some NTs have large spikes on the PES where high TPs emerge. The latter are not so well adapted. We guess that the LEPs further exist for the chains with many more atoms, so to say, the LEP is an intensive property of the FK model, i.e. independent on the size of the chain. However, a proof of this conjecture seems far from straight forward. The existence of the LEP with a barrier of less than 2 energy units is a hint of a possible 'superlubricity' of the corresponding one-dimensional chain. It is a positive contrast to the assumption that such superlubricity is possible only for 2-dimensional on-site potentials and chains [81]. But note that our understanding of superlubricity does not mean a frictionless one. It means that the friction of the chain is much lower than one would assume with a first knowledge of high energy SPs of the PES, and high TPs of an NT.

The second, downstream problem is that the NT of interest can be found with its corresponding search direction. Every stationary point is connected with some neighbours by NTs, however, one usually does not know the corresponding search direction of the NT. In our cases we search a special connection between the global minimum and the pre-SP, from there to the intermediate minimum, from there to the post-SP, and then to the next recurrence of the global minimum. In many cases we have a unique NT which connects all these points. In other cases we have two different search directions for two NTs for two parts of the pathway. But in all cases we have a nice symmetry of the energy profiles for the two parts of the pathways. The symmetry allows us to assume that really a symmetric relation between kinks and anti-kinks takes place along the LEP of interest. The case of two different NTs may be problematic for a tilting with the force direction in one of both possibilities. Usually one of the parts of the path then is enforced by the external force, however, the other part can be inhibited by the tilting.

\section{Supplementary materials}

The authors confirm that the data supporting the findings of this study are available within the article and its supplementary materials, see the appendix.

\section{Disclosure statement}

No potential conflict of interest was reported by the authors.

\section{Funding}

We acknowledge the financial support from the Spanish Ministerio de Economía y Competitividad, Project: CTQ2016-76423-P,

Spanish Structures of Excellence María de Maeztu program through grant MDM-20170767,

and from the Generalitat de Catalunya, Departament

d'Empresa i Coneixement, Project: 2017 SGR 348. 


\section{References}

1. O.M. Braun, Y.S. Kivshar, Phys. Rep. 306, 1 (1998)

2. J. Tekić, P. Mali, The ac driven Frenkel-Kontorova model (University of Novi Sad, Novi Sad, 2015)

3. D. Gangloff, A. Bylinskii, I. Counts, W. Jhe, V. Vuletić, Nat. Phys. 11, 915 (2015)

4. L. Karpa, A. Bylinskii, D. Gangloff, M. Cetina, V. Vuletić, Phys. Rev. Lett. 111, 163002 (2013)

5. O. Braun, A. Naumovets, Surf. Sci. Rep. 60, 79 (2006)

6. A. Vanossi, O.M. Braun, J. Phys.: Condens. Matter 19, 305017 (2007)

7. G. Grüner, Rev. Mod. Phys. 60, 1129 (1988)

8. R. Thorne, Physics Today 1996, 42 (1996)

9. I. Garcia-Mata, O.V. Zhirov, D.L. Shepelyansky, Eur. Phys. J. D 41, 325 (2007)

10. J.P. Pouget, C. R. Physique 17, 332 (2016)

11. O. Braun, T. Dauxois, M.V. Paliy, M. Peyrard, Phys. Rev. B 54, 321 (1996)

12. E.G. Ekomasov, R.R. Murtazin, O.B. Bogomazova, A.M. Gumerov, J. Magnetism Magnetic Materials 339, 133 (2013)

13. L. Trallori, Phys. Rev. B 57, 5923 (1998)

14. W. Que, M.B. Walker, Phys. Rev. B 46, 14772 (1992)

15. R.C. Dinsmore III, M.H. Bae, A. Bezryadin, Appl. Phys. Lett. 93, 192505 (2008)

16. M.H. Bae, R.C. Dinsmore III, T. Aref, M. Brenner, A. Bezryadin, Nano Lett. 9, 1889 (2009)

17. R. Besseling, R. Niggebrugge, P.H. Kes, Phys. Rev. Lett. 82, 3144 (1999)

18. N. Kokubo, R. Besseling, V.M. Vinokur, P.H. Kes, Phys. Rev. Lett. 88, 247004 (2002)

19. O. Iaroshenko, V. Rybalko, V.M. Vinokur, L. Berlyand, Sci. Rep. 3, 1758 (2013)

20. O.V. Zhirov, G. Casati, D.L. Shepelyansky, Phys. Rev. E 65, $026220(2002)$

21. S. Watanabe, H.S.J. van der Zant, S.H. Strogatz, T.P. Orlando, Physica D 97, 429 (1996)

22. A.V. Ustinov, M. Cirillo, B.A. Malomed, Phys. Rev. B 47, 8357 (1993)

23. A. Vanossi, N. Manini, M. Urbakh, S. Zapperi, Rev. Mod. Phys 85, 529 (2013)

24. N. Manini, O.M. Braun, E. Tosatti, R. Guerra, A. Vanossi, J. Phys. 28, 134006 (2016)

25. W. Quapp, J.M. Bofill, Molec. Phys. 117, 1541 (2019)

26. W. Quapp, J.M. Bofill, European Phys. J. B 92, 95 (2019)

27. W. Quapp, J. Theoret. Comput. Chem. 2, 385 (2003)

28. O.M. Braun, B. Hu, A. Filippov, A. Zeltser, Phys. Rev. E 58, $1311(1998)$

29. J. Tekić, D. He, B. Hu, Phys. Rev. E 79, 036604 (2009)

30. I. Sokolović, P. Mali, J. Odavić, S. Radosevic, S.Y. Medvedeva, A.E. Botha, Y.M. Shukrinov, J. Tekic, Phys. Rev. E 96, 022210 (2017)

31. M. Weiss, F.J. Elmer, Z. Physik B Cond. Matter 69, 55 (1997)

32. J. Odavic, P. Malik, J. Tekic, Phys. Rev. E 91, 052904 (2015)

33. M. Hirano, K. Shinjo, Phys. Rev. B 41, 11837 (1990)

34. A. Socoliuc, R. Bennewitz, E. Gnecco, E. Meyer, Phys. Rev. Lett. 92, 134301 (2004)

35. M. Dienwiebel, G.S. Verhoeven, N. Pradeep, J.W.M. Frenken, J.A. Heimberg, H.W. Zandbergen, Phys. Rev. Lett. 92, 126101 (2004)
36. E. Gnecco, S. Maier, E. Meyer, J. Phys.: Cond. Mat. 20, 354004 (2008)

37. E. Meyer, E. Gnecco, Friction 2, 106 (2014)

38. A. Bylinskii, D. Gangloff, V. Vuletić, Sci. 348, 1115 (2015)

39. O.M. Braun, Surface Sci. 230, 262 (1990)

40. S.R. Sharma, B. Bergersen, B. Joos, Phys. Rev. B 29, 6335 (1984)

41. Y. Braiman, J. Baumgarten, J. Jortner, J. Klafter, Phys. Rev. Lett. 65, 2398 (1990)

42. S.L. Shumway, J.P. Sethna, Phys. Rev. Lett. 67, 995 (1991)

43. C. Baesens, R.S. MacKay, Nonlinearity 11, 949 (1998)

44. T. Strunz, F.J. Elmer, Phys. Rev. E 58, 1601 (1998)

45. N. Theodorakopoulos, M. Peyrard, R.S. MacKay, Phys. Rev. Lett. 93, 258101 (2004)

46. I.D. Mikheikin, M.Y. Kuznetsov, E.V. Makhonina, V.S. Pervov, J. Mater. Synth. Process. 10, 53 (2002)

47. A. Bylinskii, D. Gangloff, I. Counts, V. Vuletić, Nature Materials 15, 717 (2016)

48. P. Bak, Rep. Prog. Phys. 45, 587 (1982)

49. C. Baesens, R.S. MacKay, Nonlinearity 17, 567 (2004)

50. Z. Zheng, B. Hu, G. Hu, Physical Review B 58, 5453 (1998)

51. O.M. Braun, A.R. Bishop, J. Röder, Phys. Rev. Let. 79, 3692 (1997)

52. O.M. Braun, H. Zhang, B. Hu, J. Tekic, Phys. Rev. E 67, $06602(2003)$

53. A.B. Kolton, D. Dominguez, N. Gronbech-Jensen, Phys. Rev. Lett. 86, 4112 (2001)

54. B. Hu, J.Y. Zhu, Phys. Rev. E 65, 016202 (2001)

55. S. Slijepčević, Chaos 25, 083108 (2015)

56. J. Odavic, P. Malik, J. Tekic, M. Pantic, M. PavkovHrvojevic, Commun. Nonlinear Sci. Numer. Simulat. 47, $100(2017)$

57. H. Li, S. Liu, Discr. Dyn. Nat. Soc. 47, 7081804 (2018)

58. O. Braun, T. Dauxois, M. Paliy, M. Peyrard, B. Hu, Physica D 123, 357 (1998)

59. W. Quapp, M. Hirsch, O. Imig, D. Heidrich, J. Comput. Chem. 19, 1087 (1998)

60. W. Quapp, M. Hirsch, D. Heidrich, Theor. Chem. Acc. 100, 285 (1998)

61. J.M. Bofill, J.M. Anglada, Theor. Chem. Acc. 105, 463 (2001)

62. R. Crehuet, J.M. Bofill, J.M. Anglada, Theor. Chem. Acc. 107, 130 (2002)

63. W. Quapp, J.M. Bofill, Theoret. Chem. Acc. 135, 113 (2016)

64. W. Quapp, J.M. Bofill, J. Ribas-Ariño, J.Phys.Chem.A 121, 2820 (2017)

65. M. Hirsch, W. Quapp, J. Molec. Struct., THEOCHEM 683, 1 (2004)

66. M. Weiss, F.J. Elmer, Phys. Rev. B 53, 7539 (1996)

67. S. Slijepčević, Nonlinearity 26, 2051 (2013)

68. J. Kiethe, R. Nigmatullin, D. Kalincev, T. Schmirander, T.E. Mehlstäubler, Nature Commun. 8, 15364 (2017)

69. T.S. van Erp, Frenkel-Kontorova models on quasi periodic potentials, Title master thesis (1999)

70. A. Vanossi, N. Manini, E. Tosatti, PNAS 109, 16429 (2012)

71. S.N. Coppersmith, Phys. Rev. A 36, 3375 (1987)

72. A. Benassi, M. Ma, M. Urbakh, A. Vanossi, Sci. Rep. 5, 16134 (2015)

73. M. Ma, A. Benassi, A. Vanossi, M. Urbakh, Phys. Rev. Lett. 114, 055501 (2015)

74. L.M. Floria, J.J. Mazo, Adv. Phys. 45, 505 (1996) 
75. A. Sadeghi, Phys. Rev. B 98, 075407 (2018)

76. S.V. Dmitriev, L.V. Nauman, A.M. Wusatowska-Sarnek, M.D. Starostenkov, phys. stat. sol. (b) 201, 89 (1997)

77. J. Zhang, X. Chen, R. Chen, L. Nie, Z. Zheng, Eur. Phys. J. B 87, $122(2014)$

78. T. Hu, K. Hu, Y. Tang, Physica B 405, 4407 (2010)

79. F. Martinez-Pedrero, P. Tierno, T.H. Johansen, A.V. Straube, Sci Rep. 6, 19932 (2016)

80. T. Pruttivarasin, M. Ramm, I. Talukdar, A. Kreuter, H. Haeffner, New J. Phys. 13, 075012 (2011)

81. C.L. Wang, W.S. Duan, Y. Yang, J.M. Chen, Commun. Theor. Phys. (Beijing, China) 54, 112 (2010)
Appendix: Supplementary Materials

The pdf-files are packed in QuBo19epjbSupplMaterials.zip

Supplementary Material $1 \mathrm{MF} 2 / 3, \mathrm{~N}=6, \ldots, 24$,

Pages 1-39, FKmodD6toD24mf2by3Suppl1.pdf

Supplementary Material $2 \mathrm{MF} 2 / 3, \mathrm{~N}=25, \ldots, 35$,

Pages 1-72, FKmodD25toD35mf2by3Suppl2.pdf

Supplementary Material 3 MF $1 / 2, N=6, \ldots, 20$, Pages 1-81, FKmodD6toD40HalfSuppl3.pdf

Supplementary Material $4 \mathrm{MF}$ reci golden mean, $\mathrm{N}=6, \ldots, 49$, Pages 1-124, FKmod6Dto49DGoldSuppl4.pdf

Supplementary Material 5 MF reci golden mean, $\mathrm{N}=50, \ldots, 69$, Pages 1-166, FKmod50Dto69DGoldSuppl5.pdf

Supplementary Material $6 \mathrm{MF} 1, \mathrm{~N}=5, \ldots, 20$, Pages 1-24, FKmodD5toD20mf1Suppl6.pdf

Throughout, node123 means a stationary point at node 123 in a calculation by an NT in a corresponding Fortran programme. SetX means a set or calculated point in a calculation by the Mathematica programme. 